\title{
A Spin-Statistics Theorem for Composites Containing both Electric and Magnetic Charges
}

\author{
John L. Friedman ${ }^{1 \star}$ and Rafael D. Sorkin ${ }^{2 \star \star}$ \\ ${ }^{1}$ Physics Department, University of Wisconsin, Milwaukee, Wisconsin \\ ${ }^{2}$ Enrico Fermi Institute, University of Chicago, Chicago, Illinois, USA
}

\begin{abstract}
The present paper states and proves an asymptotic spin-statistics theorem for composites consisting of electrically and magnetically charged particles. We work in the framework of a nonrelativistic theory, taking as the classical configuration space a $U(1)$ bundle over the space of physical configurations, and as the quantum hilbert space the homogeneous square integrable functions on that bundle. The theorems are proved using a formalism we develop here for treating "gauge spaces" - $U(1)$ bundles with connections; in particular, two products related to tensor products of vector bundles prove to be extremely useful in displaying the structure of the gauge spaces that naturally arise in this theory.
\end{abstract}

\section{Introduction}

This paper is a sequel to one in which we formulated a first-quantized theory of a system of electric and magnetic charges interacting (non-relativistically) through their instantaneous force-fields [1]. As the culmination of that paper, which we call here "I", we described - in special cases - the mechanisms by which both the spin-type (integer or half-integer) of a dyonic composite and concomitantly its asymptotic statistics (even- or oddness of the wave function under dyon interchange) can be in effect the reverse of those given by the usual combination rules (see also $[2,3,9]$ ). We then sketched, and promised to prove, a more general result which first would clarify in what sense dyons do behave in the asymptotic limit just like ordinary particles whose degrees of freedom split up into internal and external ones so that their spin and statistics can be reliably defined, and second would confirm from this point of view that the above mentioned reversals occur precisely when they "ought to". The present paper undertakes these tasks.

In order to express and prove our results most clearly, we have to redescribe the bundle of $I$ in a coordinate free way, which unfortunately relies on a much larger body of mathematics than figures in I. Rather than obscure the simple

* Supported in part by the National Science Foundation under grant number PHY 77-07111

$\star \star$ Supported in part by the National Science Foundation under grant number PHY 78-24275 
outlines of our basic arguments, we have omitted from the main text most of the mathematical preparation. To make up for this we include two Appendices in which the various definitions and facts we need are systematically presented. Because of the need to redefine most of the basic constructs, the present paper will be virtually self contained. In a few places our present notation differs from that of I, but we have tried to point out all such differences as they occur.

The paper proceeds as follows : In Sect. II we construct the configuration space $\mathbb{E}$ of a system of $e$-poles and $g$-poles (electric and magnetic charges). $\mathbb{E}$ is a $U(1)$ bundle with connection - a "gauge space" - over the space of physical configurations of the particles, and it is constructed as a certain product ( $\otimes)$ of the gauge spaces corresponding to $e$-pole-g-pole pairs. The euclidean group (of rotations and translations of $\mathbb{R}^{3}$ ) and the group of like particle permutations are then realized as groups of connection preserving automorphisms of $\mathbb{E}$. In Sect. III, restricting consideration to a system of $N$ identical $e$-poles and $N$ identical $g$-poles, we show that under certain circumstances the system can be regarded as consisting of $N e-g$ composites (dyons). Namely, in the asymptotic limit in which dyon separation is much larger than dyon size, the configuration space $\mathbb{E}$ becomes isomorphic to a gauge space $\tilde{\mathbb{E}}$ in which the external and internal degrees of freedom of the $N$ dyons are cleanly separated; formally, we introduce a second product $(\bowtie)$ which lifts to gauge spaces the notion of cartesian product of manifolds and then show that $\tilde{\mathbb{E}}$ is the $\otimes$-product of a trivial gauge space representing the center of mass positions of the $N$ dyons and of $N 1$-dyon gauge spaces representing the internal degrees of freedom of each dyon. Realizations of the euclidean group and the permutation group on $\tilde{\mathbb{E}}$ are induced by the corresponding asymptotic actions on $\mathbb{E}$. In Sect. IV we observe that the representation of $\tilde{\mathbb{E}}$ as a $\otimes$-product of gauge spaces corresponds at the hilbert space level to a tensor product of hilbert spaces which again represent the external and internal dyon degrees of freedom. The realizations of the euclidean and permutation groups on $\tilde{\mathbb{E}}$ induce representations on the quantum hilbert space of (homogeneous) functions on $\tilde{\mathbb{E}}$, and we find that a reversal of spin type and of statistics occurs in dyon formation precisely when the electromagnetic angular momentum of a dyon is a half-integral multiple of $\hbar$. For bosonic constituents, for example, the representation of the euclidean group $\mathscr{E}$ is doubled valued - i.e. is a faithful representation of the double covering group $\overline{\mathscr{E}}$ - if and only if the interchange of two dyons is represented (on the quantum hilbert space over $\tilde{\mathbb{E}}$ ) by $(-1)$; and both occur if and only if $e g=\left(n+\frac{1}{2}\right) \hbar$. Finally, in Sect. V we state the generalization of these results to composites containing arbitrary numbers of particles each having in general both electric and magnetic charge.

Finally, we would like to mention that for apparently very different reasons, the procedure known as "geometric" or "Kostant-Souriau" quantization $[4,5]$ deals with mathematical objects closely similar to what we are calling "gauge-spaces", and many of the results of that theory are useful here. Physically the difference between "geometric quantization" and what we have done seems to be that whereas they deal with a $U(1)$ bundle over phase space and introduce their connection $A$ in relation to the symplectic structure of that space, our $U(1)$ bundle is an enlarged configuration space into which we introduce a connection to describe the velocity dependent interaction between $e$-poles and $g$-poles. 
Nevertheless it is possible that the two approaches are more closely related than the above would make them seem. At a minimum one could ask whether, by subjecting to geometric quantization the classical version of our theory (as described in Sect. 3 of I) one would produce the same quantum-mechanical hilbert space and Hamiltonian operator as obtained in Sect. 4 of I and in its Appendix.

\section{The Gauged Configuration Space and Its Symmetries}

Definition. A gauge-space $\mathbb{E}=(E, M, \pi, \Gamma, A)$ is a principal $U(1)$-bundle with bundle space $E$, base manifold $M$, projection $\pi: E \rightarrow M$, and $U(1)$-action $\Gamma$, together with a connection given by a normalized equivariant 1 -form $A$ on $E$.

Accordingly,

Definition. A gauge-space morphism $T: \mathbb{E}^{\prime} \rightarrow \mathbb{E}^{\prime \prime}$ is a continuous map $T: E^{\prime} \rightarrow E^{\prime \prime}$ commuting with the action of $U(1)$ [i.e. $\left.T \circ \Gamma^{\prime}(u)=\Gamma^{\prime \prime}(u) \circ T \forall u \in U(1)\right]$ and such that the pullback $\left.T^{*}\left(A^{\prime \prime}\right)\right)$ of $A^{\prime \prime}$ by $T$, coincides with $A^{\prime}$.

If $T^{-1}$ exists and is also a gauge-space morphism then $T$ is an isomorphism and $\mathbb{E}^{\prime}$ and $\mathbb{E}^{\prime \prime}$ are isomorphic $\left(\mathbb{E}^{\prime} \simeq \mathbb{E}^{\prime \prime}\right)$. $T$ is then an automorphism if $\mathbb{E}^{\prime}=\mathbb{E}^{\prime \prime}$; we denote by "Aut($(\mathbb{E})$ " the group of all such $T$ 's. If $T$ is any gauge-space morphism then the continuous map between base spaces, $t: M^{\prime} \rightarrow M^{\prime \prime}$ given by $t \circ \pi^{\prime}=\pi^{\prime \prime} \circ T$ is said to be induced by $T$ and $T$ is said to be a lift of $t$.

Remarks. (i) We will usually omit explicit mention of $\Gamma$, writing $\Gamma(u)(X)$ simply as $u X$; thus we may speak of "the gauge-space $(E, M, \pi, A)$ ".

(ii) Except where there is danger of confusion or in formal settings such as in the statement of a theorem, we will denote canonical isomorphism by "=".

(iii) We identify $U(1)$ with the unit circle $C:=\{z \in \mathbb{C}|| z \mid=1\}$.

Notice that in contrast to $I$ we here do not include any metric $g_{a b}$ in the structure of a gauge-space. Otherwise the definitions agree, although we here call the bundle-space " $E$ " rather than " $B$ ". ("Eich" in German = "gauge").

As discussed in $I$ the relation (in which " $d$ " is exterior derivative)

$$
d A=\pi^{*}(f)
$$

defines on $M$ a unique 2-form $f=\operatorname{curv}(\mathbb{E})$.

Definition. For any manifold $M$ the trivial gauge-space over $M$ is the gauge-space $E=C \times M$ with projection $(u, x) \rightarrow x, U(1)$-action $u\left(u^{\prime}, x\right)=\left(u u^{\prime}, x\right)$, and connection $A$ such that a curve $\bar{\gamma}(t)=(u(t), x(t))$ is horizontal in $E$ iff $u(t)$ is constant. A trivial realization of a group $G$ on $E$ is a realization which acts trivially on the fiber, i.e. one of the form $R=1 \times r$. It is not hard to show (see for example the Lemma of Sect. 5 of I) that any $T \in \operatorname{Aut}(C \times M)(C \times M$ trivial $)$ must be of the form

$$
T=u \times t
$$

for $u \in C$.

The configuration space of a system of $N_{e} e$-poles (of strength $e_{p}, p=1, \ldots, N_{e}$ ) and $N_{g} g$-poles (of strength $g_{\hat{q}}, \hat{q}=\hat{1}, \ldots, \hat{N}_{g}$ ) will be a gauge space, henceforth to be called $\mathbb{E}$, over the space $\mathbb{R}^{3\left(N_{e}+N_{g}\right)}$ of physical configurations of the $N_{e}+N_{g}$ particles. We will build $\mathbb{E}$ from the gauge space (denoted $\mathbb{D}_{1 / 2}$ ) appropri- 
ate to a single dyon (a pair of particles: one electric charge, $e$, and one magnetic charge, $g$ ) with $2 e g=\hbar c$; and in terms of this construction we will realize the pertinent symmetries of our system - namely, the inhomogeneous rotation group and the group of permutations of identical particles - as gauge space automorphisms of $\mathbb{E}$.

The space $\mathbb{D}_{1 / 2}=\left(D_{1 / 2}, M_{D}, \pi_{1 / 2}, \Gamma_{1 / 2}, A_{1 / 2}\right)$ (introduced in I, Sect. 3, as the bundle of a single dyon in its center of mass frame) is described in a covariant manner in Appendix A. By introducing bases for the spaces $\Sigma$ and $V^{0}$ of that appendix, we here exhibit $\mathbb{D}_{1 / 2}$ concretely, in a form which proves to be identical to that given by Trautman [6] ${ }^{1}$. To wit: $\mathbb{D}_{1 / 2}$ is a $U(1)$ bundle over the space $M_{D}$ $=\mathbb{R}^{3}-\{0\}$ of physical configurations of a dyon with fixed center of mass. It is therefore four dimensional and can be identified with the nonzero elements of $\mathbb{C}^{2}$ :

$$
D_{1 / 2}=\mathbb{C}^{2}-\{0\}
$$

so that a point of $D_{1 / 2}$ is a pair $\xi^{I}, I=0,1$, of complex numbers. With $U(1)$ identified as $C$ (the unit complex circle), one gets the action

$$
\Gamma_{1 / 2}(u) \xi^{I}=u \xi^{I}
$$

We can write $\xi^{I}$ in the form

$$
\left(\begin{array}{l}
\xi^{0} \\
\xi^{1}
\end{array}\right)=\left(\begin{array}{l}
\sqrt{r} e^{\frac{i}{2}(\chi-\phi)} \cos \theta / 2 \\
\sqrt{r} e^{\frac{i}{2}(\chi+\phi)} \sin \theta / 2
\end{array}\right)
$$

where $r=\xi^{0} \overline{\xi^{0}}+\xi^{1} \xi^{1}$ and $(\chi, \theta, \phi)$ are the Euler angles of the element of SU(2) that maps $\left(\begin{array}{c}\sqrt{r} \\ 0\end{array}\right)$ to $\left(\begin{array}{c}\xi^{0} \\ \xi^{1}\end{array}\right)$. Then the projection $\pi_{1 / 2}: D_{1 / 2} \rightarrow M_{D}$ takes $\xi^{I}$ to the point of $\mathbb{R}^{3}-\{0\}$ with spherical coordinates $(r, \theta, \phi)$. Finally, the connection $A_{1 / 2}$ is defined by

$$
\begin{aligned}
A_{1 / 2} & =\operatorname{Im}\left(\overline{\xi^{0}} d \xi^{0}+\overline{\xi^{1}} d \xi^{1}\right) /\left(\overline{\xi^{0}} \xi^{0}+\bar{\xi}^{1} \xi^{1}\right) \\
& =\frac{1}{2}(d \chi-\cos \theta d \phi)
\end{aligned}
$$

and its curvature is

$$
\frac{1}{2} \sin \theta d \theta \wedge d \phi \equiv \frac{1}{2} \stackrel{\circ}{F}
$$

in Cartesian coordinates $y^{l}$ on $\mathbb{R}^{3}$,

$$
\stackrel{\circ}{F}_{\imath \kappa}(y)=\varepsilon_{\imath \kappa \lambda} \frac{y^{\lambda}}{|y|^{3}} .
$$

To build $\mathbb{E}$ from $\mathbb{D}_{1 / 2}$ we will need to define a product of gauge spaces and also to extend to gauge spaces the notion of the pullback of a bundle. Appendix B establishes, in a series of propositions, properties of these spaces and of their

1 In fact, we differ from Trautman only in the sign of the angle $\phi$ defined below, or equivalently, in the choice of orientation for $M_{D}$ 
morphisms needed in the paper. In order to make the remaining sections more comprehensible we repeat the key definitions here.

Definition. Given a gauge space $\mathbb{E}=(E, M, \pi, \Gamma, A)$, a manifold $\hat{M}$, and a continuous map $t: \hat{M} \rightarrow M$, the pullback $\overleftarrow{t}(\mathbb{E})$ of $\mathbb{E}$ by $t$ is the gauge space $(\hat{E}, \hat{M}, \hat{\pi}, \hat{\Gamma}, \hat{A})$, where

$$
\begin{gathered}
\hat{E}=\{(X, \hat{x}) \in E \times \hat{M} \mid \pi(X)=t(\hat{x})\}, \\
\hat{\pi}(X, \hat{x})=\hat{x}, \hat{\Gamma}(u)(X, \hat{x})=(\Gamma(u)(X), \hat{x}), \text { and } \\
\hat{A}=T^{*}(A),
\end{gathered}
$$

with the map $T: \hat{E} \rightarrow E$ defined by $T(X, \hat{x})=X$.

It follows from (2.9) that the curvatures of $\mathbb{E}$ and $\overleftarrow{t}(\mathbb{E})$ are related by

$$
\operatorname{curv} t(\mathbb{E})=t^{*} \operatorname{curv}(\mathbb{E}) \text {. }
$$

Definition. If $\Phi_{1}$ and $\Phi_{2}$ are two circles acted on by $U(1)$, their tensor product $\Phi$ $=\Phi_{1} \otimes \Phi_{2}$ is the cartesian product $\Phi_{1} \times \Phi_{2}$ modulo the equivalence relation $\left(u X_{1}, X_{2}\right) \sim\left(X_{1}, u X_{2}\right), X_{i} \in \Phi_{i}, u \in U(1)$. Then $\Phi$ is itself a circle acted on by $U(1)$ via

$$
\left(u\left(X_{1} \otimes X_{2}\right)=\left(u X_{1}\right) \otimes X_{2}=X_{1} \otimes\left(u X_{2}\right) .\right.
$$

The following gauge space product $\otimes$ is akin to the tensor product of vector bundles ${ }^{2}$.

Definition. Given two gauge space $\mathbb{E}_{i}=\left(E_{i}, M_{i}, \pi_{i}, \Gamma_{i}, A_{i}\right)$, the product $\mathbb{E}_{1} \otimes \mathbb{E}_{2}$ is the gauge space $(E, M, \pi, \Gamma, A)$, where

$$
E=\left\{X_{1} \otimes X_{2} \mid X_{i} \in E_{i} \text { and } \pi_{1}\left(X_{1}\right)=\pi_{2}\left(X_{2}\right)\right\},
$$

$M=M_{1} \cap M_{2}, \pi\left(X_{1} \otimes X_{2}\right)=\pi_{1}\left(X_{1}\right)=\pi_{2}\left(X_{2}\right), \Gamma(u)\left(X_{1} \otimes X_{2}\right)=\left(u X_{1}\right) \otimes X_{2}$, and where $A$ is defined by requiring

$$
\int_{\bar{\gamma}} A=\int_{\bar{\gamma}_{1}} A_{1}+\int_{\bar{\gamma}_{2}} A_{2},
$$

for any curves $\bar{\gamma}: I \rightarrow E, \bar{\gamma}_{i}: I \rightarrow E_{i}$ such that $\forall t \bar{\gamma}(t)=\bar{\gamma}_{1}(t) \otimes \bar{\gamma}_{2}(t)$.

It follows that on $M$

$$
\operatorname{curv}\left(\mathbb{E}_{1} \otimes \mathbb{E}_{2}\right)=\operatorname{curv}\left(\mathbb{E}_{1}\right)+\operatorname{curv}\left(\mathbb{E}_{2}\right) \text {. }
$$

The generalization of the $\otimes$-product to $m$ gauge spaces $\mathbb{E}_{i}$ over base spaces $M_{i}$ is immediate; $\mathbb{E}_{1} \otimes \ldots \otimes \mathbb{E}_{m}$ is a gauge space over $M=M_{1} \cap \ldots \cap M_{m}$, and on $M$

$$
\operatorname{curv}\left(\underset{k}{\otimes} \mathbb{E}_{k}\right)=\sum_{k} \operatorname{curv}\left(\mathbb{E}_{k}\right) \text {. }
$$

Finally, we define a corresponding product of bundle isomorphisms via

Fact 2.1. Let $\mathbb{E}_{k}$ and $\mathbb{F}_{k}, k=1, \ldots, m$ be gauge spaces over $M_{k}$ and $N_{k}$, respectively; and let $T_{k}: \mathbb{E}_{k} \rightarrow \mathbb{F}_{k}$ be gauge space morphisms, compatible in the sense that the induced maps $t_{i}: M_{i} \rightarrow N_{i}$ agree on $M=M_{1} \cap \ldots \cap M_{m}:\left.t_{1}\right|_{M}=\ldots=\left.t_{m}\right|_{M}$. Then

2 In fact if $V_{1}$ and $V_{2}$ are line bundles associated with principal $U(1)$ bundles $\mathbb{E}_{1}$ and $\mathbb{E}_{2}$ having the same base space, then the product bundle $V_{1} \otimes V_{2}$ is an associated bundle of $\mathbb{E}_{1} \otimes \mathbb{E}_{2}$ 
$\underset{k}{\bigotimes} T_{k}: \underset{k}{\bigotimes} \mathbb{E}_{k} \rightarrow \underset{k}{\bigotimes} \mathbb{F}_{k}$, defined by

$$
T_{1} \otimes \ldots \otimes T_{m}\left(X_{1} \otimes \ldots \otimes X_{m}\right)=T_{1}\left(X_{1}\right) \otimes \ldots \otimes T_{m}\left(X_{m}\right)
$$

is a gauge space morphism and is compatible with the $T_{k}$. (This appears as Fact B9 in Appendix B.)

We can use the property (2.14) of the $\otimes$-product to generalize $\mathbb{D}_{1 / 2}$ to a sequence of gauge-spaces $\mathbb{D}_{n / 2}$ defined over $M_{D}$ for all $n \in \mathbb{Z}$ and such that

$$
\operatorname{curv}\left(\mathbb{D}_{n / 2}\right)=\frac{n}{2} \stackrel{\circ}{F}
$$

To begin with we introduce

$$
\mathbb{D}_{-1 / 2}=\overline{\mathbb{D}}_{1 / 2} \text {, }
$$

with bundle space, base space and projection identical to those of $\mathbb{D}_{1 / 2}$, but having $\Gamma_{-1 / 2}(u) \xi=u^{-1} \xi$ and $A_{-1 / 2}=-A_{1 / 2}$ (see also Appendix A). Then $\operatorname{curv}\left(\mathbb{D}_{-1 / 2}\right)=$ $-\operatorname{curv}\left(\mathbb{D}_{1 / 2}\right)($ Eq. A 28$)$ and therefore satisfies $(2.15)$ in the case $n=-1$.

We then define

(if $n>0$ :)

$$
\mathbb{D}_{n / 2}=\mathbb{D}_{1 / 2} \otimes \ldots \otimes \mathbb{D}_{1 / 2} \quad(n \text { factors }),
$$

(if $n<0$ :)

$$
\mathbb{D}_{n / 2}=\mathbb{D}_{-1 / 2} \otimes \ldots \otimes \mathbb{D}_{-1 / 2} \quad(-n \text { factors }),
$$

(if $n=0:$ )

$$
\mathbb{D}_{0}=C \times M_{D} \quad \text { (the trivial gauge-space) } .
$$

Notice incidentally that by Facts B8 and B6(ii) the $\mathbb{D}_{n / 2}$ form a group under $\otimes$ :

$$
\mathbb{D}_{m / 2} \otimes \mathbb{D}_{n / 2}=\mathbb{D}_{(m+n) / 2} \text {. }
$$

We denote by

$$
x=\left(x_{1}, \ldots, x_{N_{e}}, x_{\hat{1}}, \ldots, x_{\hat{N}_{g}}\right)
$$

the point of $\mathbb{R}^{3\left(N_{e}+N_{g}\right)}$ representing a configuration in which the $e$-pole labelled by $p$ is at $x_{p} \in \mathbb{R}^{3}\left(p=1, \ldots, N_{e}\right)$ and the $g$-pole labelled by $\hat{p}$ is at $x_{\hat{p}}\left(\hat{p}=\hat{1}, \ldots, \hat{N}_{g}\right)$. We define also

$$
y_{p \hat{q}}=x_{p}-x_{\hat{q}},
$$

noting that $y_{p \hat{q}}$ can be regarded as a linear map from $\mathbb{R}^{3\left(N_{e}+N_{g}\right)}$ to $\mathbb{R}^{3}$ and that then the restriction of $y_{p \hat{q}}$ to

$$
M_{p \hat{q}}=\left\{x \in \mathbb{R}^{3\left(N_{e}+N_{g}\right)} \mid x_{p \hat{q}} \neq x_{\hat{q}}\right\}
$$


projects $M_{p \hat{q}}$ onto $M_{D}=R^{3}-\{0\}$. Finally we define in terms of the above

$$
\mathbb{E}=\bigotimes_{p \hat{q}} \mathbb{E}_{p \hat{q}}
$$

where

$$
\mathbb{E}_{p \hat{q}}=\overleftarrow{y}_{p \hat{q}}\left(\mathbb{D}_{n(p, \hat{q}) / 2}\right)
$$

embodies the interaction between $e_{p}$ and $g_{\hat{q}}$. Here $n(p, \hat{q})=2 e_{p} g_{\hat{q}} / \hbar c$ is necessarily an integer and " $y_{p \hat{q}}$ " really means the restriction $y_{p \hat{q}} \mid M_{p \hat{q}}$ of $y_{p \hat{q}}$ to $M_{p \hat{q}}$. From (2.20) and the definition of $\otimes$, the base manifold of $\mathbb{E}$ is

$$
M=\bigcap_{p \hat{q}} M_{p \hat{q}}=\left\{x \in \mathbb{R}^{3\left(N_{e}+N_{g}\right)} \mid \forall p, \hat{q}, x_{p} \neq x_{\hat{q}}\right\} ;
$$

and from (2.15), (2.10), and (2.14)

$$
f=\operatorname{curv}(\mathbb{E})=\sum_{p \hat{q}} \frac{1}{2} n(p, \hat{q})\left(y_{p \widehat{q}}\right)^{*}(\stackrel{\circ}{F}) .
$$

In the "Cartan notation" (2.23) becomes

$$
f=\sum_{p \hat{q}} \frac{1}{2} n(p, \hat{q}) \stackrel{\circ}{F}\left(y_{p \hat{q}}\right)_{l \kappa} d y_{p \hat{q}}^{l} \otimes d y_{p \hat{q}}^{\kappa}
$$

which coincides with Eq. (69) of $I$, so that $\mathbb{E}$ is a gauge-space with the same base manifold and curvature as that introduced in I (and called there " $G$ "). To confirm that that it is indeed the same gauge-space we could exhibit an isomorphism in terms of charts; but we should really ask more generally whether the curvature $f$ in fact suffices to characterize $\mathbb{E}$. Were this not so our theory would be ambiguous; for the construction in I (see especially Sect. 4 and Appendix A) of a quantum mechanical hilbert space and hamiltonian appropriate to a given system of $e$-and $g$-poles required only that $\mathbb{E}$ be a gauge-space over $M$ with curvature $f$. Fortunately one knows quite generally that, as long as the first homotopy group, $\pi_{1}(M)$, vanishes, any gauge-space over $M$ is given up to isomorphism by its curvature $f$. (See for example [4, Sect. 18].) And with the image in mind of a loop in $M$ as a motion of the $e$ - and $g$-poles at the completion of which each pole has resumed its original location, a little thought shows that indeed $\pi_{1}(M)=0$.

Having thus established the uniqueness of our theory, and in particular of $\mathbb{E}$, let us construct the symmetries of $\mathbb{E}$ which express respectively the homogeneity and isotropy of 3-space, and the indistinguishability of similarly charged poles.

Turning first to the (proper) euclidean group $\mathscr{E}$ of (orientation preserving ${ }^{3}$ ) rigid motions of $\mathbb{R}^{3}$, or rather to its universal covering group $\overline{\mathscr{E}}$, we seek a realization $\Lambda: \overline{\mathscr{E}} \rightarrow \operatorname{Aut}(\mathbb{E})$. Now we built $\mathbb{E}$ from $\mathbb{D}_{n / 2}$; and Appendix A supplies for (the covering group of) the rotation group the realization $R_{1 / 2}: \overline{\mathrm{SO}(3)} \rightarrow \operatorname{Aut}\left(\mathbb{D}_{1 / 2}\right)$, whose induced realization $r=r_{1 / 2}$ (acting in $M_{D}$ ) is just the covering projection of

3 Notice that spatial reflections are not symmetries of the physical system since precisely one of the quantities $e, g$ must be axial 
$\overline{\mathrm{SO}(3)}$ onto $\mathrm{SO}(3)$, if we regard the latter as being a group of homeomorphisms of $M_{D^{*}}$. In terms of the concrete $\mathbb{D}_{1 / 2}$ introduced above, $R_{1 / 2}$ is the action of $\overline{\mathrm{SO}(3)} \simeq \mathrm{SU}(2)$ on $\mathbb{D}_{1 / 2} \simeq \mathbb{C}^{2}-\{0\}$, and $r$ is the corresponding rotation of $M_{D} \simeq \mathbb{R}^{3}-\{0\}$. Then Fact (2.1) (or, precisely, Fact B12) yields a realization

$$
R_{n / 2}=R_{1 / 2} \otimes \ldots \otimes R_{1 / 2}
$$

such that $r_{n / 2}=r$. By composing $R_{n / 2}$ with the map $\varrho: \overline{\mathscr{E}} \rightarrow \overline{\mathrm{SO}(3)}$ which takes an element $\tilde{e}$ of $\overline{\mathscr{E}}$ into its "rotation part" we get a realization of $\overline{\mathscr{E}}$ in $\mathbb{D}_{n / 2}$,

$$
\Lambda_{n / 2}=R_{n / 2} \varrho \varrho,
$$

which induces in $M_{D}$ the realization

$$
\lambda_{n / 2}=\mathrm{r} \circ \varrho
$$

We now build $\Lambda$ from the realizations (2.25).

If $\tilde{e}$ is any element of $\overline{\mathscr{E}}$ and $e$ its projection into $\mathscr{E}$ then $e$ acts on $\mathbb{R}^{3\left(N_{e}+N_{g}\right)}$ (in the obvious way) by taking $x$ as given by (2.18) to

$$
\lambda(\tilde{e})(x)=\left(e\left(x_{1}\right), \ldots, e\left(x_{\hat{N}_{g}}\right)\right) .
$$

Now fix $p, \hat{q}$, let $n=n(p, \hat{q})$, and notice that $y_{p \hat{q}}$, being a difference of points of $\mathbb{R}^{3\left(N_{e}+N_{g}\right)}$, takes $\lambda \mid M_{p \hat{q}}$ into $\lambda_{n / 2}$ in the sense that

$$
y_{p \hat{q}} \circ \lambda(\tilde{e})=\lambda_{n / 2}(\tilde{e}) \circ y_{p \hat{q}}
$$

for every $\tilde{e}$ in $\overline{\mathscr{E}}$.

Then fact (B4) with the replacements $t \rightarrow y_{p \hat{q}} \mid M_{p \hat{q}}, \hat{M} \rightarrow M_{p \hat{q}}, M \rightarrow M_{D}, \mathbb{E} \rightarrow \mathbb{D}_{n / 2}$, $R \rightarrow \Lambda_{n / 2}, r \rightarrow \lambda_{n / 2}, \hat{r} \rightarrow \lambda \mid M_{p \hat{q}}, G \rightarrow \overline{\mathscr{E}}$ furnishes a realization $\Lambda_{p \hat{q}}: \overline{\mathscr{E}} \rightarrow \operatorname{Aut}\left(\mathbb{E}_{p \hat{q}}\right)$ which induces $\lambda \mid M_{p \hat{q}}$. Adopting the terminology of Appendix B and leaving implicit the restrictions of $y_{p \hat{q}}$ and $\lambda$ to $M_{p \hat{q}}$ we will write

$$
\Lambda_{p \hat{q}}=\overleftarrow{y}_{p \hat{q}}\left(\Lambda_{n / 2}\right)
$$

and call $\Lambda_{p \hat{q}}$ the "pullback of $\Lambda_{n / 2}$ over the pair $\left(\lambda, y_{p \hat{q}}\right)$ ".

Since all $\Lambda_{p \hat{q}}$ thereby defined induce the same realization on $M$ (namely $\lambda \mid M$ ) they are by definition compatible, and further recourse to Fact (B12) rewards us at last with a realization on $\mathbb{E}$,

$$
\Lambda=\underset{p \hat{q}}{\otimes} \Lambda_{p \hat{q}}
$$

We turn now to the group of like particle permutations. Let $(P, \hat{Q}) \in S_{N_{e}} \times S_{N_{g}}$ (where $S_{N}$ is the group of permutations of $N$ objects) belong to this group so that $e_{P(p)}=e_{p}, g_{\hat{Q}(\hat{q})}=g_{\hat{q}}$ for all $p, \hat{q}$. The corresponding permutation $\varpi=\varpi(P, \hat{Q})$ of particle positions on $\mathbb{R}^{3\left(N_{e}+N_{g}\right)}$ is given by ${ }^{4}$

$$
(P, \hat{Q})\left(x_{1}, \ldots, x_{\hat{1}}, \ldots\right)=\left(x_{P^{-1}(1)}, \ldots, x_{\hat{Q}^{-1}(\hat{1})}, \ldots\right) .
$$

4 In $I, P^{-1}(p)$ was written " $p P$ ". Also notice that " $\varpi$ " is a lower case pi 


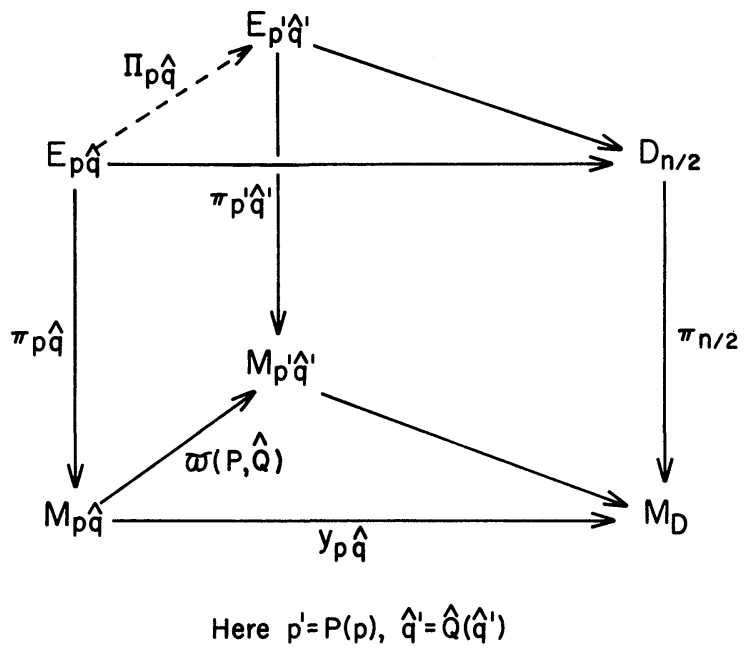

Fig. 1

We want to lift $\varpi$ to a symmetry $\Pi(P, \hat{Q})$ of $\mathbb{E}$. First note that $\varpi$ gives rise to an isomorphism $\Pi_{p \hat{q}}: \mathbb{E}_{p \hat{q}} \rightarrow \mathbb{E}_{P(p) \hat{Q}(\hat{q})}$ as follows. The relation ${ }^{5}$

$$
y_{p \hat{q}}=y_{P(p) \hat{Q}(\hat{q})}{ }^{\circ} \varpi(P, \hat{Q})
$$

corresponds to a commutative diagram on the base spaces $M_{p \hat{q}}, M_{P(p), \hat{Q}(\hat{q})}$ and $M_{D}$, as shown in Fig. 1. This diagram is a special case of Fig. 3 in which $S$ is the identity morphism, $\operatorname{id}\left(\mathbb{D}_{n / 2}\right)$. Thus, because the pullback is a functor, there is a lift

$$
\Pi_{p q}: E_{p \hat{q}} \rightarrow E_{P(p) \hat{Q}(\hat{q})}
$$

of $\varpi$ which makes Fig. 1 commute. [In the terminology of Appendix Ba) $\Pi_{p \hat{q}}$ is the pullback of $\operatorname{id}\left(\mathbb{D}_{n / 2}\right)$ over the triple $\left(\varpi, y_{P(p) \hat{Q}(\hat{q})}, y_{p \hat{q}}\right)$; the pullback is given concretely by Eq. (2.8).] Since $\varpi$ and $\operatorname{id}\left(\mathbb{D}_{n / 2}\right)$ are isomorphisms, $\Pi_{p \hat{q}}$ is an isomorphism as well.

We can now use the characterization (2.21) of $\mathbb{E}$ as a product of the $\mathbb{E}_{p \mathfrak{q}}$ 's to define the symmetry $\Pi(P, \hat{Q}) \in \operatorname{Aut}(\mathbb{E})$ as a product of the $\Pi_{p \hat{q}}$. Thus we set, for $(P, \hat{Q})$ in the group of like particle permutations

$$
\Pi(P, \hat{Q})=\underset{p \hat{q}}{\Pi_{p \hat{q}}}(P, \hat{Q}) .
$$

It then follows from the functoriality of the $\nabla$-product as discussed in Appendix B [or from Eq. (2.36) below] that the $\Pi(P, \hat{Q})$ compose in the same way as the permutations $(P, \hat{Q})$ :

$$
\Pi\left(P_{1}, \hat{Q}_{1}\right) \circ \Pi\left(P_{2}, \hat{Q}_{2}\right)=\Pi\left(P_{1} \circ P_{2}, \hat{Q}_{1} \circ \hat{Q}_{2}\right)
$$

and thus realize the group of like particle permutations.

$5 \quad$ With $x_{p}, v_{\hat{q}}$, and $y_{p \hat{q}}$ regarded as maps from $\mathbb{R}^{3\left(N_{e}+N_{g}\right)}$ to $\mathbb{R}^{3}$ the definition of $\varpi(P, \hat{Q})$ becomes $x_{p} \circ \varpi$ $=x_{P^{-1}(p)}$ or equivalently $x_{P(p)}{ }^{\circ} \varpi(P, \hat{Q})=x_{p}$ (and similarly for $\left.x_{\hat{q}}\right)$. Then [writing $\left.P(p)=p^{\prime}, \hat{Q}(\hat{q})=\hat{q}^{\prime}\right]$

$y_{p^{\prime} \hat{q}^{\prime}} \circ \varpi=x_{p^{\prime}} \circ \varpi-x_{\hat{q}^{\prime}} \circ \varpi=x_{p}-x_{\hat{q}}=y_{p \hat{q}}$,

which is $(2.31)$ 
An explicit form for $\Pi$ can be given in terms of the concrete definitions (2.8) and (2.12), (see also Fact. 2.1) of the pullback and the $\nabla$-product. According to (2.22), (2.21), and these definitions

$$
E_{p \hat{q}}=\left\{(\zeta, x) \in D_{n / 2} \times M_{p \hat{q}} \mid \pi_{n / 2}(\zeta)=y_{p \hat{q}}(x)\right\}
$$

and

$$
E=\left\{\bigotimes_{p \hat{q}}\left(\zeta_{p \hat{q}}, x\right)_{p \hat{q}} \mid\left(\zeta_{p \hat{q}}, x\right) \in E_{p \hat{q}}\right\}
$$

where the notation " $(\zeta, x)_{p \hat{q}}$ " means that $(\zeta, x)$ is the factor belonging to $\mathbb{E}_{p \hat{q}}$. Then (2.33) becomes

$$
\Pi(P, \hat{Q})\left(\bigotimes_{p \hat{q}}\left(\zeta_{p \hat{q}}, x\right)_{p \hat{q}}\right)=\bigotimes_{p \hat{q}}\left(\zeta_{p \hat{q}}, \varpi(P, \hat{Q})(x)\right)_{P(p) \hat{Q}(\hat{q})}
$$

In concluding this section let us verify that the realizations $\Lambda$ and $\Pi$ just constructed coincide with those introduced in I and on which we there based our analysis of spin and statistical reversal. For $\Lambda$, the desired coincidence follows on general grounds from the fact, demonstrated in Sect. 5 of I, that the group $\overline{\mathscr{E}}$ has at most one realization in any gauge-space.

As for $\Pi$, the automorphism $\Pi(P, \hat{Q})$ was in I (and under the name " $\bar{P}$ ") uniquely defined by requiring that it be the identity on the fiber $\pi^{-1}(x)$ over a point $x$ such that

$$
\varpi(P, \hat{Q})(x)=x
$$

But it is clear that $\Pi(P, \hat{Q})$ as given in (2.36) does have this feature. For both sides of (2.36) are in essence products of the same set of factors $\zeta_{p \hat{q}}$. If they differ, it is only because the factors belong to different base-points in $M$, which occurs precisely when (3.37) fails.

\section{The Asymptotic ("Dyonic") Configuration Space}

To define composite particles in quantum mechanics, one separates the "external" degrees of freedom corresponding to the position of the composites' centers of mass from the remaining, "internal", degrees of freedom of each composite. Ordinarily, this includes a decomposition of the configuration space into a cartesian product

$$
M^{\mathrm{ext}} \times M_{1}^{\mathrm{int}} \times \ldots \times M_{n}^{\mathrm{int}}
$$

say, of spaces representing first the center of mass positions and then the relative positions of the particles within each composite system. And the corresponding 
decomposition of the quantum mechanical hilbert space, $\mathfrak{H}$, into a tensor product

$$
\mathfrak{S} \simeq \mathfrak{H}^{\text {ext }} \otimes \mathfrak{H}_{1}^{\text {int }} \otimes \ldots \otimes \mathfrak{H}_{n}^{\text {int }},
$$

relies on the relation

$$
L^{2}(M \times N)=L^{2}(M) \otimes L^{2}(N) .
$$

In our case, however, because the configuration space $\mathbb{E}$ incorporates a gauge degree of freedom, no decomposition of the form (3.1) exists. Instead, to obtain analogous decompositions, we must first lift the notion of cartesian product to a product of gauge spaces (which we will call “ $₫$ "). Then, recalling from I that the quantum hilbert space here is the space $L_{h}^{2}(\mathbb{E})$ of homogeneous square integrable functions on $\mathbb{E}$

$$
L_{h}^{2}(\mathbb{E})=\left\{\psi \in L^{2}(\mathbb{E}) \mid \psi(u X)=u \psi(X)\right\},
$$

we must - and do - find that

$$
L_{h}^{2}(\mathbb{E} \otimes \mathbb{F})=L_{h}^{2}(\mathbb{E}) \otimes L_{h}^{2}(\mathbb{F}) .
$$

Let us therefore define the new product, $\bowtie$ :

Definition. Given two gauge space $\mathbb{E}_{i}=\left(E_{i}, M_{i}, \pi_{i}, \Gamma_{i}, A_{i}\right)$, the product $\mathbb{E}_{1} \bowtie \mathbb{E}_{2}$ is the gauge space $(E, M, \pi, \Gamma, A)$, where

$$
\begin{aligned}
E & =\left\{X_{1} \otimes X_{2} \mid X_{i} \in E_{i}\right\}, \\
M & =M_{1} \times M_{2},
\end{aligned}
$$

$\pi\left(X_{1} \otimes X_{2}\right)=\left(\pi\left(X_{1}\right), \pi\left(X_{2}\right)\right), \Gamma(u)\left(X_{1} \otimes X_{2}\right)=\left(u X_{1}\right) \otimes X_{2}$, and where $A$ is (again) defined by requiring

$$
\int_{\bar{\gamma}} A=\int_{\frac{\gamma_{1}}{1}} A_{1}+\int_{\frac{\gamma_{2}}{2}} A_{2},
$$

for any curves $\bar{\gamma}: I \rightarrow \mathbb{E}, \bar{\gamma}_{i}: I \rightarrow \mathbb{E}_{i}$ such that $\bar{\gamma}(t)=\bar{\gamma}_{1}(t) \otimes \bar{\gamma}_{2}(t) \forall t \in I$.

The extension to a product of $m$ gauge spaces is obvious. Properties of $\bowtie$ needed in the text are established in Appendix B, and we repeat here only two facts.

Fact 3.1. Let $\mathbb{E}_{i}, i=1, \ldots, m$, be gauge spaces over $M_{i}$ and let $p_{i}: M_{1} \times \ldots \times M_{m} \rightarrow M_{i}$ be the canonical projections. Then

$$
\mathbb{E}_{1} \otimes \ldots \otimes \mathbb{E}_{m} \simeq \overleftarrow{p}_{1}\left(\mathbb{E}_{1}\right) \otimes \ldots \otimes \overleftarrow{p}_{m}\left(\mathbb{E}_{m}\right)
$$

Fact 3.2. If $\mathbb{E}_{i}$ and $\mathbb{F}_{i}, i=1, \ldots, m$ are gauge spaces and $T_{i}: \mathbb{E}_{i} \rightarrow \mathbb{F}_{i}$ are morphisms, then $\underset{i}{凶} T_{i}: \mathbb{i}_{i} \mathbb{E}_{i} \rightarrow \underset{i}{凶} \mathbb{F}_{i}$, given by

$$
T_{1} \otimes \ldots \otimes T_{m}\left(X_{1} \otimes \ldots \otimes X_{m}\right)=T_{1}\left(X_{1}\right) \otimes \ldots \otimes T_{m}\left(X_{m}\right),
$$

is a morphism. 


\section{a) Definition and Symmetries}

Let us assume henceforth (until Sect. V) that $N_{e}=N_{g}=N$ and that all the $e$-poles [resp. $g$-poles] have the same mass, $m_{e}$ [resp. $m_{g}$ ] and the same charge $e$ [resp. $g$ ]; then for all $p, \hat{q} n(p, \hat{q})=2 e g / \hbar c=: n$. We assume without loss of generality that $n>0$ (the case $n=0$ is trivial).

In this section we want to show that there is an asymptotic region in which the $2 N$ poles pair off into $N e$ - $g$-composites or "dyons" which behave like "ordinary" particles with kinematically decoupled degrees of freedom. To this end we will define a gauge-space, $\tilde{\mathbb{E}}$, with respect to which the dyons behave precisely in this way and then argue that the full gauge-space $\mathbb{E}$ becomes isomorphic to $\tilde{\mathbb{E}}$ as the ratio of dyon size to inter-dyon separation goes to zero.

As in $I$ we form the $N$ dyons by arbitrarily pairing off the $2 N$ poles in the manner $\{1, \hat{1}\},\{2, \hat{2}\}, \ldots,\{N, \hat{N}\}$, and let, for $P$ any permutation of $\{1 \ldots N\}, \hat{P}$ be the corresponding permutation of $\{\hat{1} \ldots \hat{N}\}$. The group of like particle permutations is now the full $S_{N} \times S_{N}$ [with the first (respectively, second) factor acting on the $e$ poles (respectively, $g$-poles)], and its realization $\Pi$ induces a realization $\Pi_{D}: S_{N} \rightarrow \operatorname{Aut}(\mathbb{E})$ of the dyon permutation group via

$$
\Pi_{D}(P)=\Pi(P, \hat{P}) \text {. }
$$

We will derive the asymptotic (or "dyonic") gauge-space from $\mathbb{E}$ by omitting from (2.21) the factors which couple the dyons to each other. Thus we define

$$
\begin{aligned}
\tilde{\mathbb{E}} & =\bigotimes_{p} \mathbb{E}_{p \hat{p}}, \\
\mathbb{E}^{\mathrm{res}} & =\bigotimes_{p \neq q} \mathbb{E}_{p \hat{q}},
\end{aligned}
$$

so that

$$
\mathbb{E}=\tilde{\mathbb{E}} \otimes \mathbb{E}^{\mathrm{res}},
$$

where the "residual" gauge-space (3.11) accounts for that part of the structure of $\mathbb{E}$ due to inter-dyon couplings. Corresponding to this decomposition of $\mathbb{E}$ there are decompositions of the realizations $\Lambda$ and $\Pi$. For $\Lambda$ we merely factor (2.29) as

with

$$
\Lambda=\tilde{\Lambda} \otimes \Lambda^{\text {res }}
$$

$$
\begin{aligned}
\tilde{\Lambda} & =\bigotimes_{p} \Lambda_{p \hat{p}} \\
\Lambda^{\mathrm{res}} & =\bigotimes_{p \neq q} \Lambda_{p \hat{q}} .
\end{aligned}
$$

Similarly, in the case of $\Pi_{D}$ we decompose, for any $P \in S_{N}$, the expression (2.33) as [see (3.9)]

$$
\Pi_{D}(P)=\tilde{\Pi}(P) \otimes \Pi^{\mathrm{res}}(P)
$$

where

$$
\begin{aligned}
\tilde{\Pi}(P) & =\bigotimes_{p} \Pi_{p \hat{p}}(P, \hat{P}) \\
\Pi^{\mathrm{res}}(P) & =\bigotimes_{p \neq q} \Pi_{p \hat{q}}(P, \hat{P}) .
\end{aligned}
$$


Observe that the last two expressions are in fact well-defined as automorphisms of $\tilde{\mathbb{E}}$ and $\mathbb{E}^{\text {res }}$ respectively since $(P, \hat{P})$ acts the same on the hatted and the unhatted indices. By (3.16) and Fact B 12 we have decomposed $\Pi_{D}$ in the form

$$
\Pi_{D}=\tilde{\Pi} \otimes \Pi^{\mathrm{res}}
$$

with $\tilde{\Pi}$ and $\Pi^{\text {res }}$ given by (3.17) and (3.18) respectively.

We turn now to the study of the dyonic gauge-space $\tilde{\mathbb{E}}$. In order to expose its structure let us define $M^{\text {ext }}=\mathbb{R}^{3 N}$ and over it the trivial gauge-space (as defined in Sect. 2)

$$
\mathbb{E}^{\mathrm{ext}}=C \times \mathbb{R}^{3 N},
$$

introducing as well

$$
M^{\text {int }}=M_{D} \times \ldots \times M_{D} \quad(N \text { factors })
$$

and the gauge-space over it

$$
\mathbb{E}^{\text {int }}=\mathbb{D}_{n / 2} \bowtie \ldots \bowtie \mathbb{D}_{n / 2} \quad(N \text { factors }) .
$$

We will also need the projection $\tilde{y}: \tilde{M} \rightarrow M^{\text {int }}$ which takes $x \in \tilde{M}$ to the $N$-tuple $\left(y_{1}(x), \ldots, y_{N \hat{N}}(x)\right)$ and the projection $\tilde{z}: \tilde{M} \rightarrow M^{\text {ext }}$ which takes $x \in \tilde{M}$ to the $n$-tuple $\left(z_{1}(x), \ldots, z_{N}(x)\right)$, where for each configuration $x \in \mathbb{R}^{6 N}$,

$$
z_{p}=z_{p}(x)=\left(m_{e} x_{p}+m_{g} x_{\hat{p}}\right) /\left(m_{e}+m_{g}\right)
$$

is the center of mass of the $p^{\text {th }}$ dyon.

\section{Lemma}

$$
\tilde{\mathbb{E}} \simeq \mathbb{E}^{\mathrm{ext}} \otimes \mathbb{E}^{\mathrm{int}}
$$

Proof. The correspondence

$$
\left(x_{p}, x_{\hat{q}}\right) \leftrightarrow\left(z_{p}, y_{q \hat{q}}\right)
$$

identifies $\tilde{M}$ to $M^{\text {ext }} \times M^{\text {int }}$ and given this identification the restrictions $\tilde{y}_{p \hat{p}}=y_{p \hat{p}} \mid \tilde{M}$ are just the projections from $\tilde{M}$ to the $M_{D}$ in (3.21). Thus $\left(\tilde{z}, \tilde{y}_{1}, \ldots, \hat{y}_{N \hat{N}}\right)$ are the projections of $\tilde{M}=M^{\text {ext }} \times M_{D} \times \ldots \times M_{D}$ onto its factors. Now $\tilde{z}\left(\mathbb{E}^{\text {ext }}\right)$, being the pullback of a trivial gauge-space, is itself trivial (Fact B3). Hence Fact B6(ii) allows $\tilde{\mathbb{E}}$ as defined by (3.10) and (2.22) to be put in the form

$$
\tilde{\mathbb{E}}=\overleftarrow{\widetilde{z}}\left(\mathbb{E}^{\mathrm{ext}}\right) \otimes\left(\bigotimes_{p} \overleftarrow{\tilde{y}}_{p \hat{p}}\left(\mathbb{D}_{n / 2}\right)\right)
$$

which by virtue of Fact 3.1 becomes

$$
\mathbb{E}=\mathbb{E}^{\mathrm{ext}} \bowtie\left(\frac{\mathrm{x}}{p} \mathbb{D}_{n 2}\right)
$$

as asserted.

The lemma just proved effects a decomposition into internal and external variables and we want to show that the realizations $\tilde{\Lambda}$ and $\tilde{\Pi}$ decompose correspondingly. Beginning with the former, we can substitute (2.28) into (3.14) to get (using also the definitions of $\otimes$ and $\tilde{y}$ )

$$
\tilde{\Lambda}=\bigotimes_{p} \overleftarrow{y}_{p \hat{p}}\left(\Lambda_{n / 2}\right)
$$


Defining further $\Lambda^{\text {ext }}$ as the trivial realization $1 \times \lambda^{\text {ext }}$, where $\lambda^{\text {ext }}(\tilde{e})$ takes $z_{p}$ to $e\left(z_{p}\right)$, and $\tilde{z}\left(\Lambda^{\text {ext }}\right)$ as the pullback of $\Lambda^{\text {ext }}$ over the pair $(\lambda, \tilde{z})$, and referring to the Remarks following Fact B4 and Fact B13 gives $\tilde{\Lambda}$ in the form corresponding to (3.26)

$$
\tilde{\Lambda}=\overleftarrow{\widetilde{z}}\left(\Lambda^{\mathrm{ext}}\right) \otimes\left[{\underset{p}{p}}_{p} \overleftarrow{\tilde{y}}_{p}\left(\Lambda_{n / 2}\right)\right] \text {. }
$$

Finally, because of Fact 3.1 and the functoriality of pullback and $\otimes$ (the consequence of which could be called "Fact 3.1 in morphism guise") there corresponds to (3.27)

$$
\begin{aligned}
& \tilde{\Lambda}=\Lambda^{\mathrm{ext}} \text { 冈 } \Lambda^{\mathrm{int}} \\
& \Lambda^{\text {int }}=\Lambda_{n / 2} \text { 冈 .. 凶 } \Lambda_{n / 2} \quad(N \text { factors }) .
\end{aligned}
$$

For $\tilde{\Pi}$ the situation is roughly analogous. We define a trivial realization on $\mathbb{E}^{\text {ext }}$

$$
\Pi^{\mathrm{ext}}=1 \times \varpi^{\mathrm{ext}}
$$

where $\varpi^{\mathrm{ext}}(P)\left(z_{1}, \ldots, z_{N}\right)=\left(z_{P^{-1}(1)}, \ldots, z_{P^{-1}(N)}\right)$. We also define on $\mathbb{E}^{\text {int }}$ the realization $\Pi^{\text {int }}$ which permutes the factors $\mathbb{D}_{n / 2}$ of (3.22) in the sense that it takes the element of $\mathbb{E}^{\text {int }}$

$$
X_{1} \otimes \ldots \otimes X_{N} \quad \text { to } \quad X_{P^{-1}(1)} \otimes \ldots \otimes X_{P^{-1}(N)} .
$$

[i.e. $\Pi^{\text {int }}(P)=\frac{\bigotimes}{p} T_{p}$ where $T_{p}$ is the identity map of the $p^{\text {th }}$ factor onto the $P(p)^{\text {th }}$ factor in (3.22) $]$. Noting that the pullback, $\overleftarrow{y}\left(\Pi^{\text {int }}\right)$, of $\Pi^{\text {int }}$ over the pair $(\varpi(P), \tilde{y})$ (as defined in Fact B4) is just $\tilde{\Pi}$ [as follows for example from the concrete forms (3.30), (2.36) (or rather its $p=q$ part) and (B1); see also (B8)] and that $\overleftarrow{\tilde{z}}\left(\Pi^{\text {ext }}\right)$ and $\tilde{y}\left(\Pi^{\text {int }}\right)$ both induce $\varpi$ in $\tilde{M}$ we can, as before, invoke the Remarks following Facts B4 and B13 to get in turn

$$
\tilde{\Pi} \simeq \overleftarrow{\tilde{z}}\left(\Pi^{\mathrm{ext}}\right) \otimes \overleftarrow{\hat{y}}\left(\Pi^{\mathrm{int}}\right)
$$

whence

$$
\tilde{\Pi}=\Pi^{\text {ext }} \bowtie \Pi^{\text {int }}
$$

$\Pi^{\text {ext }}$ and $\Pi^{\text {int }}$ may be said to permute, respectively, the external and internal dyon degrees of freedom.

\section{b) Asymptotic Isomorphism With $\mathbb{E}$}

We saw in $I$ that a certain sub-gauge-space of $\mathbb{E}$ in which the internal dyon degrees of freedom were frozen out becomes trivial in a certain asymptotic region. We are now prepared to generalize this by demonstrating that $\mathbb{E}^{\text {res }}$ is asymptotically trivial, from which it will follow that $\mathbb{E}$ and $\tilde{\mathbb{E}}$ are asymptotically isomorphic. Then the decomposition (3.24), (3.28), (3.31) of dyon variables into internal and external (as opposed to the suppression of the former in $I$ ) will make asymptotic sense in $\mathbb{E}$ and will thereby give meaning also to the notions of asymptotic dyon spin and statistics. 
In order to render our notation of "asymptotic" more precise let us arbitrarily fix a minimum separation between dyons ${ }^{6}, l$, and define for $0 \leqq \varepsilon<\infty$

$$
\left(\mathbb{R}^{6 N}\right)_{\varepsilon}=\left\{x \in \mathbb{R}^{6 N} \mid \max \left(l, \max _{p}\left\|y_{p \hat{p}}\right\|\right) \leqq \varepsilon \min _{p \neq q}\left\|z_{p}-z_{q}\right\|\right\} .
$$

For small $\varepsilon,\left(\mathbb{R}^{6 N}\right)_{\varepsilon}$ is a set of configurations in which dyon diameters are much smaller than dyon separations; we will refer to this as the "dyonic regime" and to the $\varepsilon \rightarrow 0$ limit as the "dyonic limit". For any gauge-space $\mathbb{E}^{\prime}$ such that $M^{\prime} \subset \mathbb{R}^{6 N}$ we define the sub-gauge-space $\mathbb{E}_{\varepsilon}^{\prime}$ by setting $M_{\varepsilon}^{\prime}=M^{\prime} \cap\left(\mathbb{R}^{6 N}\right)_{\varepsilon}$ and $E_{\varepsilon}^{\prime}=\pi^{\prime-1}\left[M_{\varepsilon}^{\prime}\right]$ [equivalently $\mathbb{E}_{\varepsilon}^{\prime}=\overleftarrow{j}\left(\mathbb{E}^{\prime}\right)$ where $j$ is the inclusion of $M_{\varepsilon}^{\prime}$ into $M$ ]. Similarly for morphisms $T^{\prime}$ and realizations $R^{\prime}$ whose induced maps on base-manifolds leave $M_{\varepsilon}^{\prime}$ invariant, we define the restrictions $T_{\varepsilon}^{\prime}$ and $R_{\varepsilon}^{\prime}$.

For gauge-spaces $\mathbb{E}^{\prime}, \mathbb{E}^{\prime \prime}$ such that $M^{\prime}, M^{\prime \prime} \in \mathbb{R}^{6 N}, \mathbb{E}^{\prime} \sim \mathbb{E}^{\prime \prime}$ will mean that for $\varepsilon \rightarrow 0$ there is a bundle isomorphism $T(\varepsilon): E_{\varepsilon}^{\prime} \rightarrow E_{\varepsilon}^{\prime \prime}$ which becomes an exact gauge-space isomorphism in the dyonic limit. [By this we mean that for sufficiently small $\varepsilon$, $T(\varepsilon)$ is a $U(1)$-bundle isomorphism and that $\forall \delta>0 \exists \varepsilon_{0}>0$ such that $\varepsilon<\varepsilon_{0} \Rightarrow\left\|T(\varepsilon)^{*}\left(A_{\varepsilon}^{\prime \prime}\right)-A_{\varepsilon}^{\prime}\right\|<\delta$. Here the argument of $\|\cdot\|$ (call it " $\Delta A^{\prime \prime}$ ) is the difference of two connections and therefore the lift of a unique 1-form $\Delta a$ on $M_{\varepsilon}^{\prime} \subset \mathbb{R}^{6 N}$, and $\|\Delta A\|$ can be taken to be the largest absolute valued assumed by any cartesian component of $\Delta a$.] Similarly $R^{\prime} \sim R^{\prime \prime}$ will mean that as $\varepsilon \rightarrow 0, T(\varepsilon)$ carries $R^{\prime}$ over into $R^{\prime \prime}$.

In order to produce an asymptotic isomorphism $\mathbb{E} \sim \tilde{\mathbb{E}}$ we write, by virtue of Fact B6(ii),

$$
\tilde{\mathbb{E}}_{\varepsilon}=\tilde{\mathbb{E}}_{\varepsilon} \otimes\left(C \times \tilde{M}_{\varepsilon}\right)
$$

and compare this with

$$
\mathbb{E}_{\varepsilon}=\tilde{\mathbb{E}}_{\varepsilon} \otimes \mathbb{E}_{\varepsilon}^{\mathrm{res}}
$$

[from (3.12)]. In view of (2.14) it is plain that an asymptotic equivalence

$$
\mathbb{E}^{\mathrm{res}} \sim C \times M^{\mathrm{res}}
$$

will imply that

$$
\mathbb{E} \sim \tilde{\mathbb{E}} \text {. }
$$

To establish (3.33) it suffices in turn to prove an exact isomorphism for $\varepsilon=0$. [Strictly speaking we should, because $\mathbb{E}_{0}^{\text {res }}$ is not compact, estimate the deviation of $\mathbb{E}_{\varepsilon}^{\text {res }}$ from $\mathbb{E}_{0}^{\text {res }}$ for small $\varepsilon$; but this was done in effect in I (see Sect. $7 b$ ) thereof) and $\Delta a$ shown to be $O(\varepsilon / l)$.] Thus we are reduced to proving

Lemma. $\mathbb{E}_{0}^{\text {res }}$ is a trivial gauge-space:

$$
\mathbb{E}_{0}^{\mathrm{res}} \simeq C \times M_{0}^{\mathrm{res}}
$$

6 We need $l$ only to exclude from the dyonic limit configurations in which separations between dyons go to zero. Without such an exclusion, and with the definition of asymptotic isomorphism used below, our proof of (3.33) would fail 
Proof. From the Definition (3.11) of $\mathbb{E}^{\mathrm{res}}$, we have

$$
\mathbb{E}_{0}^{\mathrm{res}}=\left(\underset{p \neq q}{\bigotimes} \mathbb{E}_{p \hat{q}}\right)_{0}=\underset{p \neq q}{\bigotimes}\left(\mathbb{E}_{p \hat{q}}\right)_{0} .
$$

This can be rearranged as

$$
\mathbb{E}_{0}^{\mathrm{res}}=\underset{p<q}{\bigotimes} \mathbb{E}_{0}^{\mathrm{res}}(p, q),
$$

where by definition

$$
\mathbb{E}_{0}^{\mathrm{res}}(p, q)=\left(\mathbb{E}_{p \hat{q}}\right)_{0} \otimes\left(\mathbb{E}_{q \hat{p}}\right)_{0} .
$$

We claim this last gauge-space is already trivial. To verify this, notice first that for $x \in M_{0}^{\mathrm{res}}, y_{p \hat{q}}=y_{p q}=-y_{q p}=-y_{q \hat{p}}$ so that, on $M_{0}^{\mathrm{res}}$,

$$
y_{q \hat{p}}=\theta \circ y_{p \hat{q}}
$$

where $\theta: M_{D} \rightarrow M_{D}$ is inversion in the origin $(\theta(x)=-x)$. Hence inserting the Definition (2.22) of $\mathbb{E}_{p \hat{q}}$ into (3.37) and using Facts B2 and B11 gives

$$
\begin{aligned}
\mathbb{E}_{0}^{\mathrm{res}}(\mathrm{p}, \mathrm{q}) & =\left[\overleftarrow{y}_{p \hat{q}}\left(\mathbb{D}_{n / 2}\right) \otimes \overleftarrow{y}_{q \hat{p}}\left(\mathbb{D}_{n / 2}\right)\right]_{0} \\
& =\left[\overleftarrow{y}_{p \hat{q}}\left(\mathbb{D}_{n / 2} \otimes \overleftarrow{\theta}\left(\mathbb{D}_{n / 2}\right)\right)\right]_{0} .
\end{aligned}
$$

Finally we can reduce the argument of $\overleftarrow{y}_{p \hat{q}}$ to a trivial gauge-space as follows by appealing in turn to (2.17a) and Fact B11, to (A29), to Fact B 8, and finally to Fact B6(ii) [see also (2.17c),d))]:

$$
\begin{aligned}
\mathbb{D}_{n / 2} \otimes \overleftarrow{\theta}\left(\mathbb{D}_{n / 2}\right) & =\bigotimes_{1}^{n}\left(\mathbb{D}_{1 / 2} \otimes \overleftarrow{\theta}\left(\mathbb{D}_{1 / 2}\right)\right) \\
& \simeq \underset{1}{\otimes}\left(\mathbb{D}_{1 / 2} \otimes \mathbb{D}_{-1 / 2}\right) \\
& =\underset{1}{\otimes}\left(C \times M_{D}\right) \\
& =C \times M_{D} .
\end{aligned}
$$

Hence (3.38) becomes by Fact B3

$$
\mathbb{E}_{0}^{\text {res }}(p, q)=C \times M_{0}^{\text {res }},
$$

as claimed, whence likewise the triviality of (3.36) as required.

With the proof of (3.33) we are prepared to discuss asymptotic dyon spin and statistics. But before concluding this section let us summarize its main conclusions in a series of asymptotic decompositions of $\mathbb{E}, \Lambda$, and $\Pi_{D}$.

For the gauge-spaces themselves we had in (3.12), (3.24). (3.22)

$$
\begin{aligned}
\mathbb{E} & =\tilde{\mathbb{E}} \otimes \mathbb{E}^{\text {res }} \\
\tilde{\mathbb{E}} & =\mathbb{E}^{\text {ext }} \otimes \mathbb{E}^{\text {int }} \\
\mathbb{E}^{\text {int }} & =\mathbb{D}_{n / 2} \bowtie \ldots \bowtie \mathbb{D}_{n / 2} \quad(N \text { factors }) .
\end{aligned}
$$

7 The second equality is an example of the type of relation whose proof we are usually leaving implicit but which could be derived rigorously using facts from Appendix B; in this case it follows from Fact B11 and the definition of $(\cdot)_{\varepsilon}$ as a pullback 
Corresponding to these we found in (3.13) (3.28), and (3.29) for the realizations of $\overline{\mathscr{E}}$,

$$
\begin{aligned}
\Lambda & =\tilde{\Lambda} \otimes \Lambda^{\mathrm{res}} \\
\tilde{\Lambda} & =\Lambda^{\mathrm{ext}} \otimes \Lambda^{\mathrm{int}} \\
\Lambda^{\mathrm{int}} & =\Lambda_{n / 2} \otimes \ldots \bigotimes \Lambda_{n / 2}
\end{aligned}
$$

and in (3.19), (3.31), and (3.30) for the realizations of $S_{N}$,

$$
\begin{aligned}
\Pi_{D} & =\tilde{\Pi} \otimes \Pi^{\mathrm{res}} \\
\tilde{\Pi} & =\Pi^{\mathrm{ext}} \otimes \Pi^{\mathrm{int}} \\
\Pi^{\mathrm{int}} & =\text { "permutation of the factors of }(3.44 \mathrm{~b}) " .
\end{aligned}
$$

Finally we found in (3.33)

$$
\mathbb{E}^{\mathrm{res}} \sim C \times M^{\mathrm{res}}
$$

without however proving any corresponding facts for $\Lambda^{\text {res }}$ or $\Pi^{\text {res }}$.

\section{The Persistence of the Spin-Statistics Connection}

\section{a) Quantum Interpretation of Previous Results}

The last section showed in effect that in the dyonic regime - and with respect to $\tilde{\mathbb{E}}$, $\tilde{\Lambda}$, and $\tilde{\Pi}$ - our $N$ dyons behave kinematically as particles whose external and internal degrees of freedom can be entirely disentangled, the only unfamiliar feature being the involvement of a gauge degree of freedom in the internal dyon configuration space, $\mathbb{D}_{n / 2}$. In this way the notions of dyon spin and statistics become unambiguous and correspond, as we will shortly confirm, to the realizations $\tilde{\Lambda}$ and $\tilde{\Pi}$.

For the constituent $e$ - and $g$-poles these notions are not so clearcut. Nonetheless the realizations $\Lambda$ and $\Pi$ are physically natural (as discussed in I) and will provide unambiguous quantum definitions of total (linear and) angular momentum and of $e$ - and $g$-pole statistics. To study how the spin and statistics of a dyon relate to the spin and statistics of its constituents amounts then to studying the relation of the above realizations to each other.

Before proceeding with this study let us complete our formal framework by introducing the quantum mechanical hilbert spaces - and associated unitary representations of $\overline{\mathscr{E}}, S_{N} \times S_{N}$, and $S_{N}$ - with respect to which questions of spin and statistics can be properly posed. Although this "quantization" is logically necessary, it proves to be but a mechanical translation which, at least for our purposes here, adds nothing to the already classically significant structures introduced in Sects. 2 and 3.

As in effect given in I this "translation" converts gauge-spaces into hilbert spaces and realizations into unitary representations. Specifically if $\hat{\mathbb{E}}$ is a gauge- 
space and $\hat{\mathbb{R}}: G \rightarrow \operatorname{Aut}(\hat{\mathbb{E}})$ a realization, then we define ${ }^{8}$ the hilbert space

$$
L_{h}^{2}(\hat{\mathbb{E}})=\left\{\psi \in L^{2}(\hat{E}) \mid \forall X \in \hat{E}, \forall u \in C, \psi(u X)=u \psi(X)\right\}
$$

and therein the unitary representation $\hat{\mathbf{R}}: G \rightarrow \operatorname{Aut}\left(L_{h}^{2}(\hat{\mathbb{E}})\right)$ given by

$$
\hat{\mathbf{R}}(g) \cdot \psi=\psi \circ \hat{R}\left(g^{-1}\right)
$$

for $g \in G$ and $\psi \in L_{h}^{2}(\hat{\mathbb{E}})$. [In Appendix B, $\hat{\mathbf{R}}$ is denoted " $L_{h}^{2}(\hat{R})$ " to emphasize the "functorial" aspect of quantization.]

For our full system of $2 \mathrm{Ne}$-poles and $g$-poles, quantization produces a hilbert space $L_{h}^{2}(\mathbb{E})$ and unitary representations $\boldsymbol{\Lambda}$ and $\boldsymbol{\Pi}$ describing respectively the quantum mechanical rotation and translation operators and the physically natural $e$ - and $g$-pole permutation operators. All this assumes however that the $e$ and $g$-poles are in themselves spinless. In order to relax this restriction we could augment $L_{h}^{2}(\mathbb{E})$ to

$$
L_{h}^{2}(\mathbb{E}) \otimes\left[\bigotimes_{1}^{N}\left(\mathfrak{H}_{e} \otimes \mathfrak{H}_{g}\right)\right]
$$

with $\mathfrak{H}_{e}$ (resp. $\mathfrak{S}_{g}$ ) being the internal ("spin”) hilbert space for a single $e$-pole (resp. $g$-pole), and correspondingly extend $\boldsymbol{\Lambda}, \boldsymbol{\Pi}$ to unitary representations on (4.3). But since the extra degrees of freedom thereby introduced would never interact with the gauge-space degrees of freedom, their spin and statistics would only combine in the usual way with each other and also with those of the gauge-space degrees of freedom. Rather than augment our hilbert spaces, then, we will stick with $L_{h}^{2}(\mathbb{E})$ and merely augment our final results to the general case of $e$ - and $g$-poles with spin.

Now from the asymptotic equivalence (3.34) it follows that asymptotically

$$
L_{h}^{2}(\mathbb{E}) \sim L_{h}^{2}(\tilde{\mathbb{E}}) .
$$

But $\tilde{\mathbb{E}}$ decomposes as in Sect. 3, and we claim that to describe $L_{h}^{2}(\mathbb{E})$ from the point of view of (4.4) is to describe our system as a collection of $N$ kinematically decoupled particles, or "dyons".

Theorem 1. The "dyonic hilbert space" $L_{h}^{2}(\tilde{\mathbb{E}})$, to which $L_{h}^{2}(\mathbb{E})$ is asymptotically equivalent, can be written in the standard form

$$
L_{h}^{2}(\tilde{\mathbb{E}}) \simeq\left(\bigotimes_{1}^{N} \mathfrak{H}_{D}\right) \otimes L^{2}\left(\mathbb{R}^{3 N}\right)
$$

with the first (resp. second) factor representing the internal (resp. external) dyon degrees of freedom. The internal or "spin" hilbert space $\mathfrak{H}_{D}$ is $L_{h}^{2}\left(\mathbb{D}_{n / 2}\right)$ with the associated unitary representation of $\overline{\mathrm{SO}(3)}$ being $\mathbf{R}_{n / 2}$ (see (2.24)) and given this, $\tilde{\Lambda}$ and $\tilde{\boldsymbol{\Pi}}$ are respectively the standard action of $\overline{\mathscr{E}}$ and the standard particle permutation group in an $N$-particle hilbert space.

8 In order that $L^{2}(\hat{\mathbb{E}})$ make sense, $\hat{M}$ must be endowed with a volume element (from which one on $\hat{\mathbb{E}}$ follows immediately). Since for us $\hat{M}$ is always a subset of $\mathbb{R}^{k}$ (for $k=3,3 N$ or $6 N$ ) we can always use the volume element induced therefrom. (This is equivalent to - but not quite the same as - what was done in $I$ ) 
Proof. According to Facts B14 and B15 a $\otimes$-product at the gauge-space level translates into a tensor product at the quantum level so that (3.44), (3.46), and (3.48) give rise respectively to ${ }^{9}$

$$
L_{h}^{2}(\tilde{\mathbb{E}})=L_{h}^{2}\left(\mathbb{E}^{\text {ext }}\right) \otimes\left[\bigotimes_{1}^{N} L_{h}^{2}\left(\mathbb{D}_{n / 2}\right)\right]
$$

corresponding to which

$$
\tilde{\Lambda}=\boldsymbol{\Lambda}^{\mathrm{ext}} \otimes\left[\bigotimes_{1}^{N} \boldsymbol{\Lambda}_{n / 2}\right]
$$

and

$$
\tilde{\boldsymbol{\Pi}}=\boldsymbol{\Pi}^{\mathrm{ext}} \otimes \boldsymbol{\Pi}^{\mathrm{int}}
$$

where

$$
\boldsymbol{\Pi}^{\text {int }}=\text { "canonical permutation of the last } N \text { factors in (4.6)". }
$$

Because of the Definition (2.25) of $\Lambda_{n / 2}, \mathbf{R}_{n / 2}$ is indeed the internal dyon rotation group in (4.7) and we will be done if we show that

$$
L_{h}^{2}\left(\mathbb{E}^{\mathrm{ext}}\right)=L^{2}\left(M^{\mathrm{ext}}\right)
$$

and that then $\boldsymbol{A}^{\text {ext }}$ and $\boldsymbol{\Pi}^{\mathrm{ext}}$ act in the standard way by acting on the coordinates of $M^{\text {ext }}=\mathbb{R}^{3 N}$. But by definition $\mathbb{E}^{\text {ext }}, \Lambda^{\text {ext }}$, and $\Pi^{\text {ext }}$ are trivial. Then in the first place we can display the required isomorphism (4.9) as $\psi \leftrightarrow \phi$ via

$$
\psi(u, x)=u \phi(x)
$$

Given this and the definition in $\S 3$ of $\Lambda^{\text {ext }}$, for example, as $1 \times \lambda^{\text {ext }}$, it follows immediately from (4.2) that $\Lambda^{\text {ext }}\left(\tilde{e}^{-1}\right) \cdot \psi=\psi \circ\left(1 \times \lambda^{\text {ext }}(\tilde{e})\right)$ corresponds to $\phi \circ \lambda^{\text {ext }}(\tilde{e})$ as asserted.

The hilbert space $L_{h}^{2}(\tilde{\mathbb{E}})$ comprises the asymptotic states of the system which make sense in the dyonic limit just as, for example, in scattering theory a free-field fock space describes the "in" and "out" asymptotic states. Theorem 1 then suggests that we regard $\tilde{\Lambda}$ as giving the operators of dyon rotation and translation, and $\tilde{\boldsymbol{\Pi}}$ as giving the operators of dyon exchange. Indeed if we accept this interpretation of $\tilde{\boldsymbol{\Lambda}}$ then that of $\tilde{\boldsymbol{\Pi}}$ is unavoidable; for to say that a unitary group is the group of particle permutation operators for a hilbert space in the form (4.5) with linear and angular momentum given by (4.7) is precisely to say that the group acts in the way described by equations $(4.8)^{10}$.

9 Notice that, as required by Fact B14, the volume element on $\tilde{M}$ is equal under the identification (3.25) to the product element on $M^{\text {ext }} \times M_{D} \times M_{D} \times \ldots \times M_{D}$

10 Notice that the fact that the dyon position operators $z_{p}$, acting in the second factor of (4.5) and giving the centers of mass of the $e$-pole- $g$-pole pairs, are correctly permuted by $\tilde{\boldsymbol{\Pi}}$ is also a consequence of (4.5) and (4.8) 
Hence we can establish both the interpretation of $\tilde{\boldsymbol{\Lambda}}$ and that of $\tilde{\boldsymbol{\Pi}}$ by showing that the physically correct represention of $\overline{\mathscr{E}}$, namely $\boldsymbol{\Lambda}$, goes over into $\tilde{\boldsymbol{\Lambda}}$ under the asymptotic isomorphism (4.4), which we now undertake ${ }^{11}$.

Notice first that (4.4) - or rather (3.34) - is in more detail the string of equivalences

$$
\mathbb{E}=\tilde{\mathbb{E}} \otimes \mathbb{E}^{\mathrm{res}} \sim \tilde{\mathbb{E}} \otimes(C \times \tilde{M})=\tilde{\mathbb{E}} .
$$

The corresponding string which we seek to establish is

$$
\Lambda=\tilde{\Lambda} \otimes \Lambda^{\mathrm{res}} \sim \tilde{\Lambda} \otimes(1 \times \lambda)=\tilde{\Lambda},
$$

of which the first is (3.45) and the last is the Remark following Fact B 13. Finally in order to prove the central equivalence we must show that $\Lambda^{\text {res }}$ is asymptotically trivial. But the dyonic limit of $\Lambda^{\text {res }}$ is trivial:

$$
\Lambda_{0}^{\text {res }}=1 \times \lambda_{0},
$$

if only because both members of (4.13) realize $\overline{\mathscr{E}}$ and gauge-space realizations of $\overline{\mathscr{E}}$ are unique. (See I, first footnote to Sect.6.) [Alternately the analogue of the computations made below for $\Pi^{\text {res }}$ would prove (4.13) directly.] From the exact isomorphism (4.13) follows, as before, the required approximate triviality of $\Lambda^{\text {res }}$ as $\varepsilon \rightarrow 0$ :

$$
\Lambda^{\text {res }} \sim 1 \times \lambda .
$$

\section{b) Reversals of Spin and Statistics in Dyon Formation}

On the basis of the interpretations just given, we are able to deduce (asymptotic) dyon spin and statistics from those of the constituent monopoles.

Let us ask first whether the dyons' spin-type is integral of halfintegral. According to Theorem 1 this depends on the representation $\mathbf{R}_{n / 2}$, and in fact on whether the latter is faithful or not of $\overline{\mathrm{SO}(3)}$. By (4.1) and (4.2) $\mathbf{R}_{n / 2}$ is faithful iff $R_{n / 2}$ is itself faithful, which it surely is (according to Appendix A) when $n=1$. Now let $(2 \pi) \in \mathrm{SO}(3)$ be the $2 \pi$-rotation, and set $T=R_{1 / 2}(2 \pi)$. We claim $T=-1$. In fact the induced map $t: M_{D} \rightarrow M_{D}$ is by definition the identity, and then $T \in \operatorname{Aut}\left(\mathbb{D}_{1 / 2}\right)$ and $T^{2}=1 \Rightarrow T= \pm 1$; but if $T$ were +1 then $R_{1 / 2}$ would not be faithful. Hence by (2.24), $R_{n / 2}(2 \pi)=\bigotimes_{1}^{n} T=(-1)^{n}$ so that $R_{n / 2}$ is faithful precisely for odd $n$.

Inasmuch as our elementary $e$ - and $g$-poles have been treated as spinless, the ordinary rule for combining angular momenta would predict integral spin for the dyons. When $n$ is odd this naive expectation is reversed and as discussed above this reversal persists if we endow the poles with intrinsic spin of their own; that is

Theorem 2. Consider a system of $N$ identical e-poles of charge e and intrinsic spin $s_{e}$ and $N$ identical $g$-poles of charge $g$ and intrinsic spin $s_{g}$. Then a reversal of spin-type occurs in dyon formation iff $n=2 \mathrm{eg} / \hbar \mathrm{c}$ is odd.

To determine the relation of dyon statistics to the statistics of the constituent poles we will relate $\tilde{\boldsymbol{\Pi}}$ to $\boldsymbol{\Pi}_{D}$ by studying $\boldsymbol{\Pi}^{\text {res }}$. To begin with, $\boldsymbol{\Pi}$ defines the individual $e$-pole and $g$-pole statistics, with the former (latter) given by the

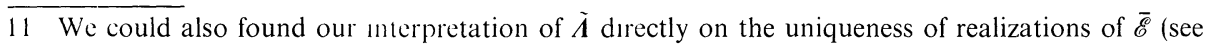
below); but the present reasoning will help prepare our discussion of dyon statistics 
behavior of $\psi \in L_{h}^{2}(\mathbb{E})$ under permutations in $S_{N} \times\{e\}\left(\{e\} \times S_{N}\right)$. Thus a wave function $\psi$ which is of pure type (i.e. either bosonic of fermionic) with respect to both kinds of poles will also be of pure type with respect to $\Pi_{D}$, and that type will follow by the usual combination rule (odd $\times$ even = odd, etc.) from the types of the $e$ - and $g$-poles. For example, a $\psi$ describing fermionic $e$-poles and bosonic $g$-poles would be odd under $\boldsymbol{\Pi}_{D}$. However it is not $\boldsymbol{\Pi}_{D}$ but $\tilde{\boldsymbol{\Pi}}$ which, according to Theorem 1, determines asymptotic dyon statistics. Just as with $\Lambda$ and $\tilde{\Lambda}, \Pi_{D}$ and $\tilde{\Pi}$ are related by (3.47); but for the group $S_{N}$ we can conclude from the asymptotic triviality of $\mathbb{E}^{\text {res }}$ only that $\Pi^{\text {res }}$ is asymptotically either trivial or the odd character of $S_{N}$. [See I, the discussion surrounding Eq. (77).]

It is precisely when $\Pi^{\text {res }}$ is not asymptotically trivial that a reversal of statistics occurs. For, writing, in correspondence to (3.49),

$$
\Pi^{\mathrm{res}} \sim \chi \times \varpi^{\mathrm{res}}
$$

where $\chi: S_{N} \rightarrow C$ is either 1 or the odd character $(-1)^{P}$, we learn from (3.47) and the definition of $\otimes$ (see Fact 2.1, also Fact B10) that there corresponds to (4.11) the chain of equivalences

$$
\Pi_{D}=\tilde{\Pi} \otimes \Pi^{\mathrm{res}} \sim \tilde{\Pi} \otimes\left(\chi \times \varpi^{\mathrm{res}}\right)=\chi \tilde{\Pi} .
$$

After quantization this becomes, in virtue of (4.1) and (4.2)

$$
\Pi_{D} \sim \chi \tilde{\Pi}
$$

so that statistics according to $\tilde{\Pi}$ agrees with statistics according to $\Pi_{D}$ precisely when $\chi \equiv 1$, that is, precisely when $\Pi^{\text {res }}$ is asymptotically trivial. We are now ready to prove

Theorem 3. A reversal of statistics occurs in dyon formation iff $n$ is odd.

Proof. As just shown a reversal fails to occur iff $\Pi^{\text {res }} \sim 1 \times \varpi^{\text {res }}$, equivalently iff $\Pi_{0}^{\text {res }}$ $=1 \times \varpi_{0}^{\text {res }}$. Thus a reversal is equivalent to $\Pi_{0}^{\text {res }}(P)=(-1) \times \varpi$, where we hereby fix $P \in S_{N}$ as the exchange $(1 \leftrightarrow 2)$, and correspondingly $\varpi$ as $\varpi_{0}^{\text {res }}(P)$. Writing for brevity $T=\Pi_{0}^{\text {res }}(P)$ and $T_{p \hat{q}}=\left(\Pi_{p q}(P, \hat{P})\right)_{0}$, we have from (3.18), the definition of $\Pi_{0}^{\text {res }}$ as a pullback, and the commutativity of pullback and $\otimes$ (Fact B11)

$$
T=\underset{p \neq q}{\bigotimes} T_{p \hat{q}}
$$

[See the discussion around (2.32) for the definition of $\Pi_{p \hat{q}}$ and $\S 3 \mathrm{~b}$ for the " ()$_{\varepsilon}$ " notation, of which " $\Pi_{0}^{\text {res" }}$ and " $\left(\Pi_{p q}(P, \hat{P})\right)_{0}$ " are instances. $]$

Equation (4.15) corresponds to (3.11) but by rearranging it suitably we can write $T$ as a product of morphisms, each of which acts on a trivial gauge-space:

$$
T=T_{12} \otimes\left(\bigotimes_{q=3}^{N} T_{q}\right) \otimes\left(\underset{2<p<q}{\otimes} T_{p q}\right)
$$

where $T_{p q}=T_{p \hat{q}} \otimes T_{q \hat{p}}$ and $T_{q}=T_{1 q} \otimes T_{2 q}$. According to (2.32), (3.37) and Fact 2.1 (or Fact B9)

$$
\begin{aligned}
& T_{p q} \in \operatorname{Aut}\left(\mathbb{E}_{0}^{\mathrm{res}}(p, q)\right) \\
& T_{q} \in \operatorname{Aut}\left(\mathbb{E}_{0}^{\mathrm{res}}(1, q) \otimes \mathbb{E}_{0}^{\mathrm{res}}(2, q)\right) .
\end{aligned}
$$


To evaluate (4.16) let us refer to the concrete expression $(2.36)$ for $\Pi(P, \hat{P})$, or rather its analogue for $T=\Pi_{0}^{\text {res }}(P)$,

$$
\bigotimes_{p \neq q}\left(\xi_{p \hat{q}}, x\right)_{p \hat{q}} \mapsto \bigotimes_{p \neq q}\left(\xi_{p \hat{q}}, \varpi(x)\right)_{p^{\prime} \hat{q}^{\prime}}
$$

[where $p^{\prime}=P(p), \hat{q}^{\prime}=\hat{P}(\hat{q})=\widehat{P(q)}$ ]. Since the the base-point $x$ plays no role in the following considerations, we can omit it and write the action of $T$ simply as

$$
\bigotimes_{p \neq q}\left(\xi_{p \hat{q}}\right)_{p \hat{q}} \mapsto \bigotimes_{p \neq q}\left(\xi_{p \hat{q}}\right)_{p^{\prime} \hat{q}^{\prime}}
$$

Now since each of the $T_{p q}, T_{q}$ is an automorphism of a trivial gauge-space over $M_{0}^{\text {res }}$, each is (2.2) of the form $u \times \varpi$ for some $u \in C$, and $T$ is therefore nontrivial iff the product of the $u$ 's is -1 . Moreover each $T_{q}$ or $T_{p q}$ permutes a finite subset of the factors of (4.18), and we will evaluate in turn each of the cases indicated by (4.16).

Consider first $T_{12} \in \operatorname{Aut}\left(\mathbb{E}_{0}^{\text {res }}(1,2)\right)$. In Sect. 3 we reduced $\mathbb{E}_{0}^{\text {res }}(1,2)$ by a succession of isomorphic replacements first [in (3.38)] to $\overleftarrow{t}\left(\mathbb{D}_{n / 2} \otimes \overleftarrow{\theta}\left(\mathbb{D}_{n / 2}\right)\right)$ where $t=\left(y_{1 \hat{2}}\right)_{0}=y_{1 \hat{2}} \mid M_{0}^{\mathrm{res}}$, and then to the trivial gauge-space (3.42). Since this succession renders $\mathbb{E}_{0}^{\text {res }}(1,2)$ explicitly trivial it must convert $T_{12}$ into an expression $u \times \varpi$ as discussed above. Moreover (3.38) and (3.39) imply (see Fact B 10) that the factor $u$ in question is $\sigma^{n}$ where $\sigma$ is the factor for $n=1$. For this case the successive replacements in question are

$$
\begin{aligned}
\overleftarrow{t}\left(\mathbb{D}_{1 / 2}\right) \otimes \stackrel{\leftarrow}{\theta \circ t}\left(\mathbb{D}_{1 / 2}\right) \\
\quad=\overleftarrow{t}\left[\mathbb{D}_{1 / 2} \otimes \overleftarrow{\theta}\left(\mathbb{D}_{1 / 2}\right)\right] \\
\simeq \overleftarrow{t}\left[\mathbb{D}_{1 / 2} \otimes \mathbb{D}_{-1 / 2}\right] \\
=\overleftarrow{t}\left[C \times M_{D}\right] .
\end{aligned}
$$

The isomorphism $\overleftarrow{\theta}\left(\mathbb{D}_{1 / 2}\right) \simeq \mathbb{D}_{-1 / 2}$ is given, according to (A30) by

$$
(\eta, v) \mapsto \overline{\Theta(\eta)}, \quad \eta \in D_{1 / 2}, \quad v \in M_{D} ;
$$

(In terms of the concrete $\mathbb{D}_{1 / 2}$ of $\S 2$,

$$
\left.\Theta(\eta)^{0}=\overline{\eta^{1}}, \quad \Theta(\eta)^{1}=-\overline{\eta^{0}}\right)
$$

and the isomorphism $\mathbb{D}_{1 / 2} \otimes \mathbb{D}_{-1 / 2}=C \times M_{D}$ is given, according to (B5) by

$$
\xi \otimes \bar{\zeta} \mapsto \xi: \zeta,
$$

where, for $\xi \propto \zeta \in \Sigma \simeq \mathbb{C}^{2}, \xi: \zeta \in C$ is the unique solution of $\xi=(\xi: \zeta) \zeta$. Then the replacements for an element of $\mathbb{E}_{0}^{\text {res }}(1,2)$ corresponding to the isomorphisms $(4.19 \mathrm{a})$ are

$$
\begin{aligned}
(\xi, x) & \otimes(\eta, x) \quad\left[\text { where } t(x)=\pi_{1 / 2}(\xi)=-\pi_{1 / 2}(\eta)\right] \\
& \mapsto(\xi \otimes(\eta, t(x)), x) \\
& \mapsto(\xi \otimes \overline{\Theta(\eta)}, x) \\
& \mapsto(\xi: \Theta(\eta), x) .
\end{aligned}
$$


Or dropping the $x$ as was done in (4.18), and consolidating, the replacement which converts $\mathbb{E}_{0}^{\text {res }}(1,2)$ to trivial form becomes finally

$$
\xi \otimes \eta \mapsto \xi: \Theta(\eta)
$$

Now by definition $[$ see $(4.18)] T_{12}(\xi \otimes \eta)=\eta \otimes \xi$. Under the replacement (4.20) this becomes

$$
\xi: \Theta(\eta) \mapsto \eta: \Theta(\xi) .
$$

But we claim that

$$
\xi: \Theta(\eta)=-\eta: \Theta(\xi)
$$

In fact if $\xi=u \Theta(\eta)$ then from the Theorem of Appendix A and the conjugate linearity of $\Theta, \Theta(\xi)=\Theta(u \Theta(\eta))=-\bar{u} \eta$, whence (since $\bar{u}=1 / u) \eta=-u \Theta(\xi)$, whence $\eta: \Theta(\xi)=-u$ as claimed. Thus (4.21) is the map -1 when $n=1$, and $T_{12}$ contributes in general a factor $(-1)^{n}$.

Consider second $T_{q}$, or for concreteness $T_{3}$. According to (4.17b), (3.37), and (4.18) this is the permutation

$$
\xi \otimes \eta \otimes \zeta \otimes \beta \mapsto \zeta \otimes \beta \otimes \xi \otimes \eta .
$$

Now with (4.20) applied to both $\mathbb{E}_{0}^{\text {res }}(1,3)$ and $\mathbb{E}_{0}^{\text {res }}(2,3)$, the LHS of $(4.23)$ reduces to $[\xi: \Theta(\eta)] \otimes[\zeta: \Theta(\beta)]$, and under the further reduction $\left(C \times M_{D}\right) \otimes\left(C \times M_{D}\right)$ $=C \times M_{D}$ [see Fact B6(ii)] it reduces further to the numerical product $[\zeta: \Theta(\eta)][\zeta: \Theta(\beta)]$. It follows that (4.23) is simply the identity map so that $T_{q}$ contributes a factor of 1 .

Finally the factors such as $T_{34}$ act as

$$
\xi \otimes \eta \mapsto \xi \otimes \eta
$$

which under the replacement (4.20) evidently amounts to nothing - or rather to 1. Thus the $T_{p q}$ for $2<p<q$ also contribute factors of 1 .

Corollary. A reversal of spin occurs in dyon formation iff a reversal of statistics occurs iff eg/hc is half-integral.

Proof. Immediate from Theorems 2 and 3.

Corollary. Dyon formation preserves the connection between spin and statistics.

\section{Summary and Final Generalization}

We began by introducing a certain gauge-space $\mathbb{E}$ to represent the degrees of freedom of a system $\mathfrak{G}$ of (spinless) point $e$-and $g$-poles; and we found natural realizations in $\mathbb{E}$ of the symmetries of $\mathbb{S}$ corresponding to translations, rotations and permutations of identical monopoles. In a certain asymptotic regime - which exists if we assume $e_{p}=e, m_{p}=m_{e}, g_{\hat{p}}=g, m_{\hat{p}}=m_{g}$, and $N_{e}=N_{g}=N-\mathbb{E}$ decomposes as a product $\mathbb{E} \sim \mathbb{E}^{\text {ext }} \otimes \mathbb{E}^{\text {int }}$ where $\mathbb{E}^{\text {ext }}=C \times \mathbb{R}^{3 N}$ and $\mathbb{E}^{\text {int }}=\mathbb{D}_{n / 2} \otimes \ldots \bowtie \mathbb{D}_{n / 2}$, so that $\subseteq$ appears as a system of $N$ composite "dyons", each with external degrees of freedom $\mathbb{R}^{3}$, as usual, and internal degrees of freedom $\mathbb{D}_{n / 2}$. 
Our realizations $\Lambda$ and $\Pi_{D}$ of $\overline{\mathscr{E}}$ and $S_{N} \subset S_{N} \times S_{N}$, respectively, agree with this asymptotic decomposition of $\mathbb{E}$ except that, when $n=2 e g / \hbar c$ is odd, $\Pi_{D}$ differs by the odd character $\chi$ from the standard form $\tilde{\Pi}$, which by definition is simple permutation of the (internal and external) dyon degrees of freedom. Moreover the gauge degree of freedom embodied by $\mathbb{D}_{n / 2}$ means that "internal" dyon angular momentum can be other than purely "orbital" and consequently can assume halfintegral values. Again this happens precisely when $n$ is odd. Interpreted in terms of the quantizations scheme of $I$, these facts mean, as we have seen, that in the "formation" of composite dyons from point $e$ - and $g$-poles (possibly with intrinsic quantum mechanical spin), a reversal of spin-type and statistics-type occurs if and only if $n$ is odd. Since the reversals always occur together, the composites obey the spin-statistics connection if the constituents do.

Within the restriction to a non-relativistic, first-quantized theory the above work falls short of full generality in three ways : the constituents are assumed to be either pure $e$-poles or pure $g$-poles; all composites are taken to be identically constituted; only dyonic pairs (as opposed to singlets, triplets, etc.) are considered. Dispensing with these limitations, one can still carry out an analysis closely similar to that of Sects. 2-4, and we conclude by sketching it.

Let there be $N$ dually charged monopoles ("point dyons") with dual charges $\left(e_{p}, g_{p}\right), p=1 \ldots N$. (We need only one sort of index now.) Define as before $M, x_{p}, y_{p q}$, and set

$$
\mathbb{E}=\underset{p<q}{\bigotimes} \mathbb{E}_{p q},
$$

where

$$
\mathbb{E}_{p q}=\overleftarrow{y}_{p q}\left(\mathbb{D}_{j(p, q)}\right)
$$

and where $j(p, q):=\left(e_{p} g_{q}-e_{q} g_{p}\right) / \hbar c$ must be of the form $n / 2$ for some integer $n$ ("Dirac quantization condition"). Just as before there are in $\mathbb{E}$ natural realizations $\Lambda$ of $\overline{\mathscr{E}}$ and $\Pi$ of the subgroup $S \subset S_{N}$ which exchanges only like monopoles.

Now let us group the point dyons in any way whatever into $K$ disjoint sets and consider as before the limit where each set forms a composite particle whose diameter is much smaller than its separation from the other composites.

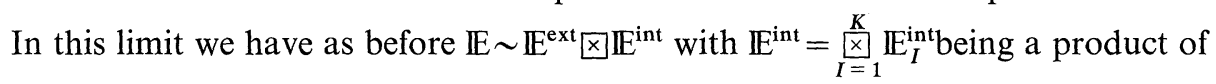
gauge-spaces each of which describes the internal degrees of freedom of one of the composites. ( $\mathbb{E}^{\text {ext }}$ is no longer trivial'however.)

Again there are natural realizations $\Lambda^{\text {ext }}, \Lambda^{\text {int }}$ of $\overline{\mathscr{E}}$ and $\Pi^{\text {ext }}, \Pi^{\text {int }}$ of the group $S_{D} \subset S$ of elements of $S$ which keep the dyonic composites together, and again

$$
\Lambda \sim \Lambda^{\mathrm{ext}} \bowtie \Lambda^{\mathrm{int}}
$$

but

$$
\Pi_{D} \sim \chi \Pi^{\mathrm{ext}} \otimes \Pi^{\mathrm{int}}
$$

where $\Pi_{D}: S_{D} \rightarrow \operatorname{Aut}(\mathbb{E})$ is the restriction of $\Pi$ to $S_{D}$, and $\chi: S_{D} \rightarrow\{ \pm 1\}$ is a character. 
For a particular composite - say the $I^{\text {th }}$ - its acquired spin is given by the action of $\overline{\mathrm{SO}(3)}$ on $\mathbb{E}_{I}^{\text {int }}$ and its acquired statistics by the sign of $\chi(P)$ for any $P \in S$ which exchanges it with another composite of like composition. The main conclusion is just as before: For a particular composite a spin reversal occurs iff a statistics reversal occurs iff, among the pairs formable from its constituent monopoles, an odd number have half-integral $j(p, q)$.

\section{Appendix A. The One-dyon, One-twist Gauge-Space}

After introducing the notion of, and notation for, $\mathrm{SO}(3)$-spinors, this appendix will construct the gauge-space $\mathbb{D}_{1 / 2}$ and the "inversion" $\Theta$, deriving in particular the equality $\Theta^{2}=-1$ to which is due the "reversal of statistics in dyon formation".

Of the many available descriptions of $\mathbb{D}_{1 / 2}([6-8]$; see also the presentation in terms of coordinate charts in I (§3)) that given in part (b) below seems to us to contain the fewest extraneous elements.

\section{a) $\mathrm{SO}(3)$-spinors}

Let $\Sigma$ be a 2-dimensional complex vector space and $\Sigma^{*}$ its dual:

$$
\Sigma^{*}=\mathscr{L}(\Sigma, \mathbb{C}) .
$$

Let $\bar{\Sigma}$ (the "conjugate" to $\Sigma$ ) be $\Sigma$ itself only with elements of $\mathbb{C}$ acting as their complex conjugates. By this we mean that any element $\xi \in \Sigma$ can be regarded at the same time as an element $\bar{\xi}$ of $\bar{\Sigma}$ and that

$$
\bar{z} \bar{\xi}=\bar{z} \bar{\xi}
$$

for all $z \in \mathbb{C}, \xi \in \Sigma$. In representing tensors formed from $\Sigma, \Sigma^{*}$, etc. we will use the "abstract index" notation so that $\xi^{A}, \xi_{A}, \xi^{A^{\prime}}, \xi_{A^{\prime}}$ are elements of $\Sigma, \Sigma^{*}, \bar{\Sigma}, \bar{\Sigma}^{*}$ respectively. (We identify $\bar{\Sigma}^{*}$ to $\overline{\Sigma^{*}}, \Sigma$ to $\Sigma^{* *}$, etc.) Thus for example, if $\xi \in \Sigma^{*}, \eta \in \bar{\Sigma}$, and $\zeta=\xi \otimes \eta \in \Sigma^{*} \otimes \bar{\Sigma}$, we write $\zeta_{A}^{B^{\prime}}=\xi_{A} \eta^{B^{\prime}}$ and express $\bar{\zeta}:=\bar{\xi} \otimes \bar{\eta}$ as $\bar{\zeta}_{A^{\prime}}^{B}=\bar{\xi}_{A^{\prime}} \bar{\eta}^{B}$.

We assume henceforth that $\Sigma$ has a distinguished positive-definite hermitian metric $G_{A^{\prime} B}$ and define its inverse $G^{A B^{\prime}}$ by

$$
G^{A B^{\prime}} G_{B^{\prime} C}=\delta_{C}^{A} \text {. }
$$

We will also write the metric, whose hermiticity appears in our notation as

$$
G_{A^{\prime} B}=\bar{G}_{B A^{\prime}},
$$

in the index-free manner

$$
\langle\xi, \eta\rangle:=\bar{\xi}^{A^{\prime}} G_{A^{\prime} B} \eta^{B} \text {. }
$$

By definition the group $\operatorname{Aut}(\Sigma ; G)$ of invertible linear operators $S^{A}{ }_{B}$ which preserve $G_{A^{\prime} B}$, i.e. such that

$$
G_{A^{\prime} A} \bar{S}_{B^{\prime}}^{A^{\prime}} S_{B}^{A}=G_{B^{\prime} B},
$$

is isomorphic (as an abstract group) to $U(2)$, and we may as well denote it by the same name. Clearly (A6) is equivalent to the condition $S^{\dagger}=S^{-1}$ where we define 
for any $S \in \mathscr{L}(\Sigma, \Sigma)$

$$
\left(S^{\dagger}\right)_{B}^{A}=G^{A A^{\prime}} \bar{S}_{A^{\prime}}^{B^{\prime}} G_{B^{\prime} B} .
$$

We will call $\Sigma$ with its distinguished hermitian metric a "space of $\mathrm{SO}(3)$ spinors" ${ }^{12}$. The relation of spinors to vectors emerges if we consider the complex vector space

$$
V=\left\{v_{B}^{A} \mid v_{A}^{A}=0\right\}
$$

and the bilinear form thereon given by

$$
(v, w)=\frac{1}{2} \operatorname{tr} v w=\frac{1}{2} v^{A}{ }_{B} w^{B}{ }_{A} .
$$

The subset of hermitian [in the sense of (A7)] elements of $V$,

$$
V^{0}=\left\{v \in V \mid v^{\dagger}=v\right\}
$$

is, together with the metric (A9), a real 3-dimensional euclidean vector space. In fact $V^{0}$ is an oriented 3 -space because setting

$$
\varepsilon\left(v_{1}, v_{2}, v_{3}\right)=\frac{1}{4 i} \operatorname{tr} v_{1}\left[v_{2}, v_{3}\right]
$$

defines canonically a 3 -form on $V^{0}$.

The prescription

$$
p(\xi)=2 \xi^{A} \bar{\xi}^{B^{\prime}} G_{B^{\prime} B}-\left(\bar{\xi}^{C^{\prime}} G_{C^{\prime} D} \xi^{D}\right) \delta^{A}{ }_{B}
$$

defines a map from $\Sigma$ onto $V^{0}$, and under this correspondence, $S \in \operatorname{Aut}(\Sigma)$ goes into the linear operator

$$
\tilde{p}(S)=\left\{v \mapsto S v S^{-1}\right\}
$$

[i.e. $\tilde{p}(S) \cdot p(\xi)=p(S \xi)=S p(\xi) S^{-1}$ ]. Plainly (A13) is a rigid rotation [it preserves the metric (A9)], and in fact the $\tilde{p}(S)$ exhaust the rotation group of $V^{0}$, which we may as well call "SO(3)". When restricted to the subgroup of $U(2)$,

$$
\mathrm{SU}(2)=\{S \in \operatorname{Aut}(\Sigma) \mid \operatorname{det} S=1\},
$$

$\tilde{p}$ expresses that $\mathrm{SU}(2)$ is the universal covering group, $\overline{\mathrm{SO}(3)}$ of $\mathrm{SO}(3)$.

In what follows we will need a 2-form (skew tensor) $\varepsilon_{A B}$ and its inverse $\varepsilon^{A B}$ defined by

$$
\varepsilon^{A C} \varepsilon_{B C}=\delta_{B}^{A} .
$$

Since $\Sigma$ is 2-dimensional such an $\varepsilon_{A B}$ is unique up to a complex factor. We can even normalize it in magnitude by requiring

$$
\varepsilon^{A B} G_{A^{\prime} A} G_{B^{\prime} B}=\varepsilon_{A^{\prime} B^{\prime}},
$$

12 One might want to include a distinguished 2-form $\varepsilon_{A B}$ in the definition of "SO(3)-spinor space". This would reduce $\operatorname{Aut}(\Sigma)$ to $\mathrm{SU}(2)$ and allow a "geometrization" (up to sign) of SO(3)-spinors 
but the phase of $\varepsilon_{A B}$ remains arbitrary. [Notice that the LHS of (A16) could be regarded as $(\operatorname{det} G) \varepsilon_{A^{\prime} B^{\prime}}$, and the positivity of $G_{A^{\prime} B}$ entails that of $\operatorname{det} G$. Thus the sign in (A16) is not arbitrary.]

Also for future use we evaluate the norm of $p(\xi)$. From (A12) and (A9) [and writing $|\xi|^{2}=\langle\xi, \xi\rangle$ and $\left.\|v\|^{2}=(v, v)\right]$

$$
\begin{aligned}
\|p(\xi)\|^{2} & =\frac{1}{2}\left(2 \xi^{A} \bar{\xi}^{B^{\prime}} G_{B^{\prime} B}-|\xi|^{2} \delta_{B}^{A}\right)\left(2 \xi^{B} \bar{\xi}^{A^{\prime}} G_{A^{\prime} A}-|\xi|^{2} \delta_{A}^{B}\right) \\
& =(2-1-1+1)|\xi|^{4}=|\xi|^{4}, \\
\|p(\xi)\| & =|\xi|^{2}=G_{A^{\prime} B} \bar{\xi}^{A^{\prime}} \xi^{B} .
\end{aligned}
$$

Finally let us relate our notation to one which might be more familiar. To this end we treat the inclusion of $V$ into $\Sigma \otimes \Sigma^{*}$ as a linear map, $\sigma$. Regarded as belonging to $V^{*} \otimes \Sigma \otimes \Sigma^{*}$, this map has the index structure $\sigma_{\alpha C}{ }^{B}$, where lower case Greek indices refer to the vector space $V$. In terms of $\sigma$ the metric (A9) on $V$ becomes

$$
g_{\alpha \beta}=\frac{1}{2} \sigma_{\alpha{ }_{B}{ }^{A}{ }_{\beta}{ }^{B}{ }_{A},},
$$

which can be used to raise and lower Greek indices, and we have then the identity $^{13}$

$$
g^{\alpha \beta} \sigma_{\alpha{ }_{B}{ }^{A}{ }_{\beta}{ }^{C}{ }_{D}}=2 \delta_{D}^{A} \delta_{B}^{C}-\delta_{B}^{A} \delta_{D}^{C} .
$$

From (A19) follows

$$
p(\xi)_{\alpha}=\bar{\xi}^{A^{\prime}} G_{A^{\prime} A} \sigma_{\alpha{ }_{B}}^{A} \xi^{B}
$$

which will look still more familiar if we define

$$
\left(\xi^{\dagger}\right)_{A}=\bar{\xi}^{A^{\prime}} G_{A^{\prime} A},
$$

and suppress the spinor indices so that (A20) appears as

$$
p(\xi)_{\alpha}=\xi^{\dagger} \sigma_{\alpha} \xi .
$$

We will also need the identity

$$
\varepsilon^{A N} \sigma_{\alpha N^{M B}}^{M}=\sigma_{\alpha A B},
$$

which follows from $\operatorname{tr} \sigma=0$ and the general relation $\varepsilon^{A B} \varepsilon_{C D}=\delta_{C}^{A} \delta_{D}^{B}-\delta_{D}^{A} \delta_{C}^{B}$.

In terms of any orthogonal basis for $\Sigma$ the metric $G_{A^{\prime} B}$ becomes the identity $2 \times 2$ matrix, $V$ becomes the set of traceless $2 \times 2$ matrices, and (A7) becomes hermitian conjugation. Hence $V^{0}$ becomes the set of traceless hermitian $2 \times 2$ matrices, and if we determine a basis for $V$ by requiring that the $\sigma_{\alpha}{ }^{A}$ be the Pauli spin matrices, we recover the standard isomorphism between $2 \times 2$ hermitian matrices and $\mathbb{R}^{3}$; under this isomorphism the 3-form $\varepsilon$ defined by (A11) becomes the usual alternating symbol for which $\varepsilon_{123}=1$.

13 To prove it use (A18) to show that the LHS of (A19) is the orthogonal projection of $\Sigma \otimes \Sigma^{*}$ onto $V$ and notice that the RHS is this same projection 


\section{b) The Gauge-space $\mathbb{D}_{1 / 2}$}

We know from I that $\mathbb{D}_{1 / 2}$ admits a faithful realization of $\overline{\mathrm{SO}(3)}$, and we have just seen that the space $\Sigma$ of $\mathrm{SO}(3)$-spinors also admits such a realization. Is it surprising then that we look to $\Sigma$ as a basis for constructing $\mathbb{D}_{1 / 2}$ ?

Definition. We imploy the notation just introduced. $\mathbb{D}_{1 / 2}=\left(D_{1 / 2}, M_{D}, \pi_{1 / 2}, \Gamma_{1 / 2}\right.$, $\left.A_{1 / 2}\right)$ is the gauge-space with $D_{1 / 2}=\Sigma-\{0\}, M_{D}=V^{0}-\{0\}, \pi_{1 / 2}=p,\left(\Gamma_{1 / 2}(u)\right)\left(\xi^{A}\right)$ $=u \xi^{A}$ for $u \in C$, and connection given by

$$
A_{1 / 2} \cdot \dot{\xi}=\operatorname{Im} \frac{\langle\xi, \dot{\xi}\rangle}{\langle\xi, \xi\rangle}
$$

where $\dot{\xi}$ is a tangent vector at $\xi \in D_{1 / 2}$.

(Because $D_{1 / 2}$ is naturally a subset of the vector space $\Sigma$ we can represent $\dot{\xi}$ as itself a vector in $\Sigma$.)

Inasmuch as we have defined $A_{1 / 2}$ in a fully $\mathrm{SO}(3)$-invariant manner, without having preferred any direction in $V^{0}$, the associated curvature $f=f_{1 / 2}$ must be sperically symmetric. Direct calculation shows

$$
f_{\alpha \beta}(v)=\frac{1}{2} \varepsilon_{\alpha \beta \gamma} v^{\gamma}\|v\|^{-3}=\frac{1}{2} \stackrel{\circ}{F}_{\alpha \beta}(v)
$$

[with $\varepsilon_{\alpha \beta \gamma}$ given by (A11)] and thereby justifies our appelation " $\mathrm{D}_{1 / 2}$ ".

Consider now the symmetries of $\mathbb{D}_{1 / 2}$. Because $\mathrm{SU}(2)$ as defined in (A14) preserves all the structure involved in defining $\mathbb{D}_{1 / 2}(\operatorname{SU}(2) \subset \operatorname{Aut}(\Sigma))$ it induces in $\Sigma-\{0\}=D_{1 / 2}$ a group of gauge-space automorphisms. Thus identifying (A14) with $\overline{\mathrm{SO}(3)}$ as discussed above gives the (by definition faithful) realization

$$
\mathbb{R}_{1 / 2}: \overline{\mathrm{SO}(3)} \rightarrow \operatorname{Aut}\left(\mathbb{D}_{1 / 2}\right)
$$

in terms of which dyon angular momentum is defined.

Although $\mathbb{D}_{1 / 2}$ admits proper rotations as symmetries, we should not expect the same for orientation reversing "rotations" of $V^{0}$ such as inversion in the origin; for the product $e g=\frac{n}{2} \hbar c$ is an axial, not a polar, scalar. Rather, if $\theta: V^{0} \rightarrow V^{0}$ is inversion in the origin $(\theta(v)=-v)$ then we should expect a lift of $\theta$ to change the sign of $A$ (and hence of the curvature). And in fact the conjugate-linear map taking $\xi^{A} \in \Sigma$ to

$$
\Theta(\xi)^{A}=\bar{\xi}^{A^{\prime}} G_{A^{\prime} B} \varepsilon^{A B}
$$

is such a lift for any $\varepsilon_{A B}$ normalized according to (A16). To see this notice first that since $\Theta$ is conjugate-linear it clearly takes fibers into fibers and reverses the action, $\Gamma_{1 / 2}$, of $U(1)$. Moreover if $\eta=\Theta(\xi)$ then by (A16) and (A4)

$$
\begin{aligned}
\eta_{A}^{\dagger} & =\bar{\eta}^{A^{\prime}} G_{A^{\prime} A}=\xi^{B} \bar{G}_{B B^{\prime}} \bar{\varepsilon}^{A^{\prime} B^{\prime}} G_{A^{\prime} A} \\
& =\xi^{B} \bar{G}_{B B^{\prime}} \bar{\varepsilon}^{A^{\prime} B^{\prime}} \bar{G}_{A A^{\prime}}=\xi^{B} \varepsilon_{A B}
\end{aligned}
$$

so that

$$
\begin{aligned}
& \eta^{\dagger}{ }_{A} \sigma_{\alpha}{ }_{B} \eta_{B} \eta^{B}=\xi^{C} \varepsilon_{A C} \sigma_{\alpha}{ }_{B} \xi^{\dagger}{ }_{D} \varepsilon^{B D} \\
& \quad=-\xi^{\dagger}{ }_{D}\left(\varepsilon^{D B} \sigma_{\alpha}{ }^{A}{ }_{B} \varepsilon_{A C}\right) \xi^{C}=-\xi^{\dagger}{ }_{D} \sigma_{\alpha}{ }^{D}{ }_{C} \xi^{C}
\end{aligned}
$$


where the final step used (A23). This confirms that $\Theta$ induces $\theta$ on $M_{D}$. Similarly one can verify for the pullback that $\Theta^{*}\left(A_{1 / 2}\right)=-A_{1 / 2}$, which is almost obvious anyway since $\Theta$ has been defined (up to a phase) solely in terms of the structure of $\mathbb{D}_{1 / 2}$.

We can also view (A26) as the linear map $\bar{\Theta}: \Sigma \rightarrow \bar{\Sigma}$ given by

$$
\xi \mapsto \overline{\Theta(\xi)} \text {. }
$$

In this sense it expresses an isomorphism of $\mathbb{D}_{1 / 2}$ and $\mathbb{D}_{-1 / 2}$ where we define

$$
\mathbb{D}_{-1 / 2}=\left(\bar{\Sigma}, M_{D}, p, \Gamma_{-1 / 2},-A_{1 / 2}\right)
$$

with $\left[\left(\Gamma_{-1 / 2}(u)\right) \bar{\xi}\right]^{A^{\prime}}=u \bar{\xi}^{A^{\prime}}=\overline{\bar{u} \xi^{A^{\prime}}}$ for $\bar{\xi} \in \bar{\Sigma}$ (recall that $\Sigma=\bar{\Sigma}$ as sets). Observe that $A_{-1 / 2}=-A_{1 / 2} \Rightarrow$

$$
\operatorname{curv}\left(\mathbb{D}_{-1 / 2}\right)=-\operatorname{curv}\left(\mathbb{D}_{1 / 2}\right) \text {. }
$$

We remark that according to its definition, $\mathbb{D}_{-1 / 2}$ coincides with the opposed gauge-space $\overline{\mathbb{D}}_{1 / 2}$ as introduced in Appendix $\mathrm{B}$. Also, since the lift $\overleftarrow{\pi}_{1 / 2}(\theta): \overleftarrow{\theta}\left(\mathbb{D}_{1 / 2}\right) \rightarrow \mathbb{D}_{1 / 2}$ (again, see Appendix B for terminology) induces on $M_{D}$ the same inversion $\theta$ as does $\Theta$, the composition

$$
\bar{\Theta} \circ \overleftarrow{\pi}_{1 / 2}(\theta): \overleftarrow{\theta}\left(\mathbb{D}_{1 / 2}\right) \rightarrow \mathbb{D}_{-1 / 2}=\overline{\mathbb{D}}_{1 / 2}
$$

is an isomorphism which induces the identity map on $M_{D}$. In terms of elements (A29) is [for $p(\xi)=-v]$

$$
(\xi, v) \mapsto \overline{\Theta(\xi)}
$$

which for fixed $v$ maps $\left[\overleftarrow{\theta}\left(\pi_{1 / 2}\right)\right]^{-1}(v)$ onto $\left(\pi_{-1 / 2}\right)^{-1}(v)$

Finally let us exhibit the crucial minus sign:

Theorem. $\Theta^{2}=-1$.

Proof. If $\eta=\Theta(\zeta)$ and $\zeta=\Theta(\eta)$ then, in light of (A26), (A21), (A27), and (A15),

$$
\zeta^{C}=\eta_{A}^{\dagger} \varepsilon^{C A}=\xi^{B} \varepsilon_{A B} \varepsilon^{C A}=-\xi^{C} .
$$

\section{Appendix B. Products and Pullbacks of Gauge-Spaces}

In this appendix we define, and collect some relevant properties of, three operations on gauge-spaces, whose explicit use greatly clarifies the relations presented in the body of the paper.

\section{a) Pullbacks}

Given a gauge-space $\mathbb{E}=(E, M, \pi, \Gamma, A)$, a manifold $\hat{M}$ and a continuous map $t: \hat{M} \rightarrow M$. one can define the pullback of $\mathbb{E}$ by $t$ as the gauge space $\hat{\mathbb{E}}$ $=(\hat{E}, \hat{M}, \hat{\pi}, \hat{\Gamma}, \hat{A})$ where $\hat{E}=\{(X, \hat{x}) \in E \times \hat{M} \mid \pi(X)=t(\hat{x})\}, \hat{\pi}(X, \hat{x})=\hat{x}$ for $(X, \hat{x}) \in \hat{E}$ and $\hat{\Gamma}(u)((X, \hat{x}))=(\Gamma(u)(X), \hat{x})$. Plainly the map $T: \hat{E} \rightarrow E$ given by $T(X, \hat{x})=X$ commutes with the action of $U(1)$ as given by $\hat{\Gamma}, \Gamma$, and we define $\hat{A}$ to make $T$ a gauge-space morphism: $\hat{A}=T^{*}(A)$. In this situation (see Fig. 2) we use the notation $\hat{\mathbb{E}}=\overleftarrow{t}(\mathbb{E})$, 


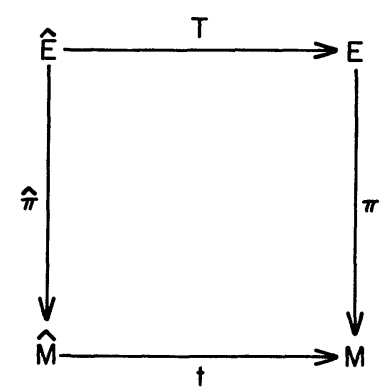

Fig. 2

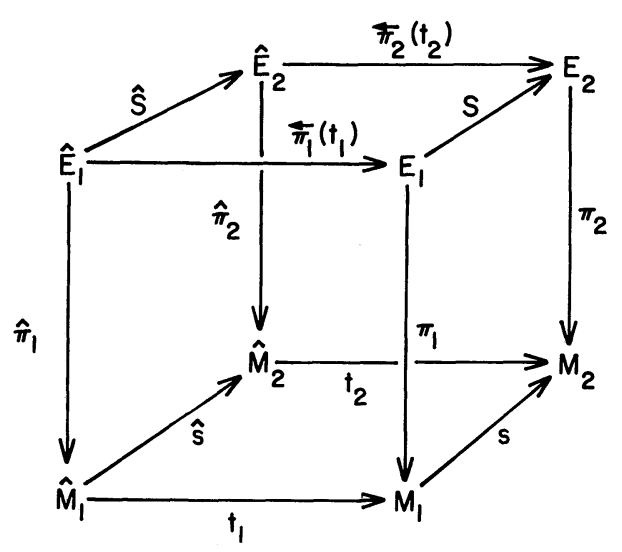

Fig. 3

$\hat{E}=\overleftarrow{t}(E), \hat{\pi}=\overleftarrow{t}(\pi), \hat{\Gamma}=\overleftarrow{t}(\Gamma), \hat{A}=\overleftarrow{t}(A)$, and $T=\overleftarrow{\pi}(t)$. Notice that $t$ is the map on basespaces induced by $T$; so our notation is consistent.

Fact B1. $\operatorname{curv}(\overleftarrow{t}(\mathbb{E}))=t^{*}(\operatorname{curv}(\mathbb{E}))$.

Proof. With notation as above and $f=\operatorname{curv}(\mathbb{E}) d \hat{A}=d T^{*}(A)=T^{*}(d A)=T^{*}\left(\pi^{*}(f)\right)$ $=(\pi \circ T)^{*}(f)=(t \circ \hat{\pi})^{*}(f)=\hat{\pi}^{*}\left(t^{*}(f)\right)$ so that $t^{*}(f)$ fulfills the Definition (2.1) of $\hat{f}=\operatorname{curv}(\hat{\mathbb{E}})$.

It is also easy to verify that

Fact B2. ${\overleftarrow{t_{1} \circ t_{2}}}_{(}(\mathbb{E}) \simeq \overleftarrow{t}_{2} \circ \overleftarrow{t}_{1}(\mathbb{E})$ (canonical isomorphism)

The notion of trivial gauge-space was defined following Eq. (2.1) and the following follows straightforwardly from the definitions.

Fact B3. The pullback of a trivial gauge-space is trivial. In the above situation with $\mathbb{E}$ the trivial gauge-space $C \times M$, the isomorphism $\hat{\mathbb{E}} \simeq C \times \hat{M}$ is given by $((u, x), \hat{x}) \leftrightarrow(u, \hat{x})$.

Let us recall from the theory of principal fiber bundles (in particular $U(1)$ bundles) another result which extends readily to gauge-spaces. To this end consider (Fig. 3) a pair of gauge-spaces $\mathbb{E}_{i}(i=1,2)$ and of maps $t_{i}: \hat{M}_{i} \rightarrow M_{i}$, and retain the notation $\overleftarrow{t}_{i}\left(\mathbb{E}_{i}\right)=\mathbb{E}_{i}$, etc. 
If " $(S, \hat{s}):\left(\pi_{1}, t_{1}\right) \rightarrow\left(\pi_{2}, t_{2}\right)$ " means that $S: \mathbb{E}_{1} \rightarrow \mathbb{E}_{2}, \hat{s}: \hat{M}_{1} \rightarrow \hat{M}_{2}$ and $s t_{1}=t_{2} \hat{S}$ (where, as always, $s$ is the map induced by $S$ ) then given $(S, \hat{s}):\left(\pi_{1}, t_{1}\right) \rightarrow\left(\pi_{2}, t_{2}\right)$ there is a unique gauge-space morphism $\hat{S}: \overleftarrow{t}_{1}\left(\mathbb{E}_{1}\right) \rightarrow \overleftarrow{t}_{2}\left(\mathbb{E}_{2}\right)$, inducing $\hat{S}$ and such that $S \circ \overleftarrow{\pi}_{1}\left(t_{1}\right)=\overleftarrow{\pi}_{2}\left(t_{2}\right) \circ \hat{S}$. We will refer to $\hat{S}$ as the "pullback of $S$ over the triple $\left(\hat{s}, t_{2}, t_{1}\right)$ ", and the use of this expression will entail that $(S, \hat{s}):\left(\pi_{1}, t_{1}\right) \rightarrow\left(\pi_{2}, t_{2}\right)$. In concrete terms $\hat{S}$ is just the function

$$
(X, \hat{x}) \mapsto(S(X), \hat{S}(\hat{x}))
$$

for $(X, \hat{x}) \in \hat{E}_{1}$.

Furthermore, if $\mathbb{E}_{3}$ is a third gauge-space and $\hat{Q}$ is the pullback of $Q: \mathbb{E}_{2} \rightarrow \mathbb{E}_{3}$ over the triple $\left(\hat{q}, t_{3}, t_{2}\right)$ then $\hat{Q} \circ \hat{S}$ is the pullback of $Q \circ S$ over the triple $\left(\hat{q} \circ \hat{S}, t_{3}, t_{1}\right)$. Finally if $\mathbb{E}_{1}=\mathbb{E}_{2}=\mathbb{E}, \hat{M}_{1}=\hat{M}_{2}=\hat{M}$ and $S=\mathrm{id}(\mathbb{E})$ and $\hat{s}=\mathrm{id}(\hat{M})$ are both identity maps, then plainly the pullback of $S$ over the trivial triple $(\hat{s}, t, t)$ is itself the identity, $\operatorname{id}(\stackrel{t}{t}(\mathbb{E}))$.

The facts recounted in the last two paragraphs can be described as the functoriality of the pullback. From this functoriality follows readily

Facts $B 4$. Let $t: \hat{M} \rightarrow M$, where $M$ is the base-manifold of a gauge-space $\mathbb{E}$. If $R, r$, $\hat{r}$, realizing the group $G$ in $\mathbb{E}, M, \hat{M}$ respectively are such that $R$ induces $r$ (as implied by the notation) and $t \circ \hat{r}=r \circ t$ (for all $g \in G$ ) then the prescription " $\hat{R}(g)=$ pullback of $R(g)$ over the triple $(\hat{r}(g), t, t)$ " lifts $\hat{r}$ to a realization $\hat{R}$ of $G$ in $\overleftarrow{t}(\mathbb{E})$, and $\overleftarrow{\pi}(t) \circ \hat{R}=R \circ \overleftarrow{\pi}(t)$

We will call $\hat{R}$ the "pullback of $R$ over the pair $(\hat{r}, t)$ ", and denote it by " $t(R)$ " (omitting reference to $\hat{r}$ ).

Remark. In Fact B4 if $\mathbb{E}=C \times M$ is trivial and $R$ is also trivial (in the sense that $R=1 \times r$ for some realization $r$ ) then $\overleftarrow{t}(R)$ is also trivial.

\section{b) Products}

If $\Phi_{i}(i=1,2)$ are gauge-space fibers [equivalently circles acted on by $U(1)$ ] then we define their tensor product $\Phi=\Phi_{1} \otimes \Phi_{2}$ to be their cartesian product $\Phi_{1} \times \Phi_{2}$ modulo the equivalence relation

$$
\left(u X_{1}, X_{2}\right) \sim\left(X_{1}, u X_{2}\right)
$$

for $u \in C$, the unit complex circle. Then $\Phi$ it itself a possible gauge-space fiber since it is topologically a circle and is acted on by $C=U(1)$ via

$$
u\left(X_{1} \otimes X_{2}\right)=\left(u X_{1}\right) \otimes X_{2},
$$

where we have written " $X_{1} \otimes X_{2}$ " for the equivalence class of $\left(X_{1}, X_{2}\right)$. Using this construction we can define two closely related types of gauge-space product.

The first of these extends to gauge-spaces the notion of cartesian product for manifolds. For an arbitrary pair $\mathbb{E}_{i}(i=1,2)$ of gauge-spaces let $\mathbb{E}=\mathbb{E}_{1} \bowtie \mathbb{E}_{2}$ be the gauge-space whose fibers are the tensor products of the fibers of $\mathbb{E}_{1}$ and $\mathbb{E}_{2}$ and whose projection is therefore the map $\pi: E \rightarrow M_{1} \times M_{2}$ given by $\pi\left(X_{1} \otimes X_{2}\right)$ $=\left(\pi_{1}\left(X_{1}\right), \pi_{2}\left(X_{2}\right)\right)$. This gives $\mathbb{E}$ as a principal $U(1)$-bundle (the definition of its topology and the proof of its local triviality being straightforward) and we define 
the connection $A$ which renders it a gauge-space by requiring

$$
\int_{\bar{\gamma}} A=\int_{\bar{\gamma}_{1}} A_{1}+\int_{\frac{\gamma_{2}}{2}} A_{2}
$$

for any curves $\bar{\gamma}: I \rightarrow E, \bar{\gamma}_{i}: I \rightarrow E_{i}$ (where $I=[0,1]$ ) such that $\forall t \bar{\gamma}(t)=\bar{\gamma}_{1}(t) \otimes \bar{\gamma}_{2}(t)$.

The second product essentially takes gauge-spaces $\mathbb{E}_{i}$ over a single $M$ and produces a gauge-space $\mathbb{E}$ over the same $M$, whose curvature is the sum of those of the $\mathbb{E}_{i}$. (In particular the Chern class of $\mathbb{E}$ will be the sum of those of the $\mathbb{E}_{i}$.) However it proves useful here to allow the base manifolds $M_{i}$ to differ and define the product only over their intersection. Thus we define for arbitrary gauge-spaces $\mathbb{E}_{1}, \mathbb{E}_{2}, \mathbb{E}=\mathbb{E}_{1} \otimes \mathbb{E}_{2}$ to be the gauge-space over $M=M_{1} \cap M_{2}$ whose fiber over $x \in M$ is the tensor product $\pi_{1}^{-1}(x) \otimes \pi_{2}^{-1}(x)$ of the fibers of $\mathbb{E}_{1}$ and $\mathbb{E}_{2}$ over $x$. In this case $\mathbb{E}$ is, so to speak, the point-wise tensor product of $\mathbb{E}_{1}$ and $\mathbb{E}_{2}$. To specify the connection on $E_{1} \otimes E_{2}$ we again use (B2) where now the $\bar{\gamma}_{i}$ (and therefore $\bar{\gamma}$ ) must be lifts of the same curve $\gamma: I \rightarrow M$.

We can summarize the two definitions as follows:

$$
\begin{aligned}
& E_{1} \otimes E_{2}=\left\{X_{1} \otimes X_{2} \mid X_{i} \in E_{i}\right\} \\
& E_{1} \otimes E_{2}=\left\{X_{1} \otimes X_{2} \mid X_{i} \in E_{i} \text { and } \pi_{1}\left(X_{1}\right)=\pi_{2}\left(X_{2}\right)\right\} .
\end{aligned}
$$

That $\otimes$ and $\otimes$ are similarly defined is apparent, and in fact either can be expressed in terms of the other using pullbacks. To express $\otimes$ in terms of $\otimes$ let $i: M_{1} \cap M_{2} \rightarrow M_{1} \times M_{2}$ be the canonical injection, $i(m)=(m, m)$. Then

$$
\mathbb{E}_{1} \otimes \mathbb{E}_{2} \simeq \overleftarrow{i}\left(\mathbb{E}_{1} \otimes \mathbb{E}_{2}\right) \quad \text { (canonically) }
$$

Conversely if $p_{i}: M_{1} \times M_{2} \rightarrow M_{i}$ are the canonical projections then

$$
\mathbb{E}_{1} \otimes \mathbb{E}_{2} \simeq \overleftarrow{p}_{1}\left(\mathbb{E}_{1}\right) \otimes \overleftarrow{p}_{2}\left(\mathbb{E}_{2}\right) \quad \text { (canonically) }
$$

Because of (B3) and (B4), a result in terms of one of the products has usually a directly equivalent form in terms of the other product. When this happens in the sequel, we will state the equivalent form only if it is needed for the body of the paper.

Fact B5. $\operatorname{curv}\left(\mathbb{E}_{1} \otimes \mathbb{E}_{2}\right)=\left.\operatorname{curv}\left(\mathbb{E}_{1}\right)\right|_{M}+\left.\operatorname{curv}\left(\mathbb{E}_{2}\right)\right|_{M}$, where $M=M_{1} \cap M_{2}$, and $\left.f\right|_{M}$ is the restriction of $f$ to $M$.

Proof. As above let $\bar{\gamma}_{1}, \bar{\gamma}_{2}$ and $\bar{\gamma}=\bar{\gamma}_{1} \otimes \bar{\gamma}_{2}$ be lifts of the same curve $\gamma$ and assume further that $\gamma$ is a loop bounding the surface $S$ in $M$ and that the curves $\bar{\gamma}_{i}$ bound lifts $\bar{S}_{i}$ of $S$ in $E_{i}$; then $\bar{\gamma}$ also bounds a surface $\bar{S}$ in $\mathbb{E}_{1} \otimes \mathbb{E}_{2}$ whose projection onto $M$ is $S$. We have

$$
\begin{gathered}
\oint_{\bar{\gamma}_{i}} A_{i}=\int_{\bar{S}_{i}} d A_{i}=\int_{S_{i}} f_{i}, \\
\oint_{\bar{\gamma}} A=\int_{\bar{S}} d A=\int_{S} f,
\end{gathered}
$$

whence from (B2)

$$
\int_{S}\left(f_{1}+f_{2}-f\right)=0 .
$$


Since $S$ is arbitrary, the integrand vanishes.

The following comes straightforwardly from the definitions:

Fact B6. Let $\mathbb{E}_{1}$ be any gauge-space over $M_{1}$ and $E_{2}=C \times M_{2}$ the trivial gaugespace over $M_{2}$, and let $p_{1}: M_{1} \times M_{2} \rightarrow M_{1}$ be the projection. Then

(i) $\mathbb{E}_{1} \otimes \mathbb{E}_{2} \simeq \overleftarrow{p}_{1}\left(\mathbb{E}_{1}\right)$ (canonically) via

$$
X_{1} \otimes\left(u, x_{2}\right) \leftrightarrow\left(u X_{1},\left(\pi_{1}\left(X_{1}\right), x_{2}\right)\right)
$$

(ii) $\mathbb{E}_{1} \otimes \mathbb{E}_{2} \simeq \mathbb{E}_{1} \mid\left(M_{1} \cap M_{2}\right)$ (canonically) via

$X_{1} \otimes(u, x) \leftrightarrow u X_{1}$.

(Here " $\mathbb{E} \mid N$ " denotes the restriction of $\mathbb{E}$ to $N \subset M$, that is $\mathbb{E} \mid N=\overleftarrow{j}(\mathbb{E})$ where $j: N \rightarrow M$ is the inclusion.)

Definition. Let $\mathbb{E}=(E, M, \pi, \Gamma, A)$. Then the opposed gauge-space is

$$
\overline{\mathbb{E}}=(E, M, \pi, \bar{\Gamma},-A) \quad \text { where } \quad \bar{\Gamma}(u) X=\Gamma\left(u^{-1}\right) X
$$

Fact B7. $\operatorname{curv}(\overline{\mathbb{E}})=-\operatorname{curv}(\mathbb{E})$.

Proof. Clear from (2.1) and the last definition.

Fact B8. $\mathbb{E} \otimes \overline{\mathbb{E}} \simeq C \times M$ (canonically).

Proof. Let $\Phi=\pi^{-1}(x)$, and $\bar{\Phi}=\bar{\pi}^{-1}(x)$. By definition both these fibers are equal as sets and we write " $\bar{X}$ " for an element of $\Phi$ regarded as belonging to $\bar{\Phi}$. Then the desired isomorphism restricted to $\Phi \otimes \bar{\Phi}$ takes $X_{1} \otimes \bar{X}_{2}$ to

$$
\psi\left(X_{1} \otimes \bar{X}_{2}\right):=\left(X_{1}: X_{2}, x\right) \in C \times M,
$$

where $X_{1}: X_{2}$ is the unique $u \in C$ for which $\Gamma(u) X_{2}=X_{1}$. The map $\psi$ will be welldefined iff for all $u \in C, \psi\left(\Gamma(u)\left(X_{1}\right) \otimes \bar{X}_{2}\right)=\psi\left(X_{1} \otimes \bar{\Gamma}(u)\left(\bar{X}_{2}\right)\right)$, which is equivalent, since $\bar{\Gamma}(u)\left(\bar{X}_{2}\right)=\overline{\Gamma\left(u^{-1}\right)\left(X_{2}\right)}$, to $\left[\Gamma(u)\left(X_{1}\right)\right]: X_{2}=X_{1}:\left[\Gamma\left(u^{-1}\right)\left(X_{2}\right)\right]$, i.e., to the relation,

$$
\Gamma(u)\left(X_{1}\right)=\Gamma(w)\left(X_{2}\right) \Leftrightarrow X_{1}=\Gamma(w) \circ \Gamma\left(u^{-1}\right)\left(X_{2}\right),
$$

whence to $\Gamma(u)^{-1} \Gamma(w)=\Gamma(w) \Gamma\left(u^{-1}\right)$, which is true for the abelian group $C$. It is also straightforward to verify, using $\bar{A}=-A$, that $\psi$ takes the connection on $\mathbb{E} \otimes \overline{\mathbb{E}}$ to the trivial connection on $C \times M$.

We consider now the $\otimes$-product of gauge-space morphisms as needed for the body of the paper. For $i=1,2$ let $\mathbb{E}_{i}\left(\right.$ resp. $\left.\mathbb{F}_{i}\right)$ be gauge-spaces over $M_{i}\left(\operatorname{resp} . N_{i}\right)$ and let $T_{i}: \mathbb{E}_{i} \rightarrow \mathbb{F}_{i}$ be morphisms such that $t_{1}\left|M=t_{2}\right| M$ where $M=M_{1} \cap M_{2}$. We will call such morphisms compatible. The obvious presription (for $X_{1} \otimes X_{2} \in E_{1} \otimes$ $E_{2}$ ),

$$
X_{1} \otimes X_{2} \mapsto T_{1}\left(X_{1}\right) \otimes T_{2}\left(X_{2}\right),
$$

defines a gauge-space morphism

$T_{1} \otimes T_{2}: \mathbb{E}_{1} \otimes \mathbb{E}_{2} \rightarrow \mathbb{F}_{1} \otimes F_{2}$, 
as is not hard to check. Moreover if $S_{i}: \mathbb{F}_{i} \rightarrow \mathbb{G}_{i}$ are also compatible morphisms then

$$
\left(S_{1} \circ T_{1}\right) \otimes\left(S_{2} \circ T_{2}\right)=\left(S_{1} \otimes S_{2}\right) \circ\left(T_{1} \otimes T_{2}\right)
$$

(functoriality). (In particular the $\otimes$-product of isomorphisms is itself an isomorphism.) More generally we can state the following self-evident result.

Fact B9. Let $\mathbb{E}_{k}$ and $\mathbb{F}_{k}$ for $k=1 \ldots m$ be gauge-spaces over $M_{k}$ and $N_{k}$ respectively; and let $\left\{T_{k}\right\}$ be a collection of compatible gauge-space morphisms $T_{k}: \mathbb{E}_{k} \rightarrow \mathbb{F}_{k}$.

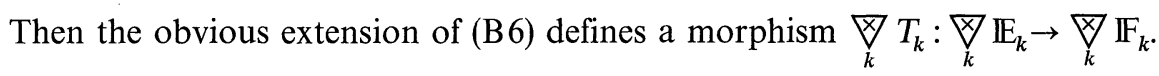

If the $T_{k}$ are all isomorphisms then so is their product.

Fact B10. For $u_{k} \in C, \underset{k}{\otimes}\left(u_{k} T_{k}\right)=\left(\prod_{j} u_{j}\right)\left(\underset{k}{\otimes} T_{k}\right)$.

In particular $u^{\prime} \otimes u^{\prime \prime}=u^{\prime} u^{\prime \prime}$

Remark. The equivalent results for $₫$ are precisely analogous, except that no compatibility of morphisms need be assumed.

Fact B11. Let $\mathbb{E}_{k}, k=1 \ldots m$ be gauge-spaces over $M_{k}$ and let $t_{k}: \hat{M}_{k} \rightarrow M_{k}$ be mutually compatible: $t_{1}\left|M=\ldots=t_{m}\right| M=t$ where $M=\cap M_{k}$. Then

$$
\bigotimes_{k} \overleftarrow{t}_{k}\left(\mathbb{E}_{k}\right) \simeq \overleftarrow{t}\left(\bigotimes_{k}^{\otimes} \mathbb{E}_{k}\right) \quad \text { (canonically) }
$$

In other words $\otimes$ commutes with pullback.

Sketch of Proof. The following is a well-defined isomorphism:

$$
\bigotimes_{k}\left(X_{k}, \hat{x}\right) \leftrightarrow\left(\bigotimes_{k} X_{k}, \hat{x}\right)
$$

where necessarily $\pi_{k}\left(X_{k}\right)=t(\hat{x})$ for all $k$.

Again there is an equivalent result for $\bowtie$.

The following are immediate corollaries of the functoriality of $\otimes$ and $\otimes$.

Fact B12. Let $\mathbb{E}_{k} k=1 \ldots m$ be gauge-spaces over $M_{k}$ and let $R_{k}: G \rightarrow \operatorname{Aut}\left(\mathbb{E}_{k}\right)$ be compatible realizations [i.e. the $R_{k}(g)$ are compatible for each $g \in G$ ]. Then $R=\underset{k}{\otimes}$ $R_{k}$ given by $R(g)=\bigotimes_{k}^{\otimes} R_{k}(g)$ realizes $G$ in $\underset{k}{\otimes} \mathbb{E}_{k}$ and is compatible with the $R_{k}$.

Fact B 13. If, for $k=1$ to $m, R_{k}$ realizes $G$ in $\mathbb{E}_{k}$ then the map $\frac{\square}{k} R_{k}$ taking $g \in G$ to $\underset{k}{\square} R_{k}(g)$ realizes $G$ in $\underset{k}{\square} \mathbb{E}_{k}$.

Remark. Let $\mathbb{E}_{1}$ and $\mathbb{E}_{2}$ be as in Fact (B6). If $R_{i}(i=1,2)$ are compatible realizations of $G$ in $\mathbb{E}_{i}$ of which the second is trivial $\left(R_{2}=1 \times r_{2}\right)$ then clearly $R_{1} \otimes R_{2}$ corresponds to $R_{1}$ under the isomorphism (ii) of Fact (B6). 


\section{c) The $\bowtie$-Product Under Quantization}

Assume henceforth that $\mathbb{E}_{1}$ and $\mathbb{E}_{2}$ are gauge-spaces whose base-manifolds $M_{1}$, $M_{2}$ are endowed with volume elements $\mu_{1}, \mu_{2}$ and let $M_{1} \times M_{2}$ have the product volume element $\mu=\mu_{1} \times \mu_{2}$. Then $\mathbb{E}_{1}$, for example, inherits a natural volume element $\bar{\mu}_{1}$ in terms of which one can define $L^{2}\left(E_{1}\right)$ and the subspace $L_{h}^{2}\left(\mathbb{E}_{1}\right)$ of $L^{2}$ functions which are homogeneous in the sense of (4.1). We have the following isomorphism (in which $\otimes$ is of course the hilbert space tensor product).

Fact B 14. $L_{h}^{2}\left(\mathbb{E}_{1} \bowtie \mathbb{E}_{2}\right) \simeq L_{h}^{2}\left(\mathbb{E}_{1}\right) \otimes L_{h}^{2}\left(\mathbb{E}_{2}\right)$ (canonically).

Proof. Let $\psi_{i} \in L_{h}^{2}\left(\mathbb{E}_{i}\right)(i=1,2)$ and let $\psi$ be the function on $E=E_{1} \bowtie E_{2}$ defined by

$$
\psi\left(X_{1} \otimes X_{2}\right)=\psi_{1}\left(X_{1}\right) \psi_{2}\left(X_{2}\right) .
$$

The homogeneity of $\psi_{1}$ and $\psi_{2}$ ensures that $\psi$ is well-defined and itself homogeneous, so that $\psi \in L_{h}^{2}(\mathbb{E})$. Moreover since the mapping $\left(\psi_{1}, \psi_{2}\right) \mapsto \psi$ is bilinear it defines a linear map $\beta_{0}$ from the algebraic tensor product of $L_{h}^{2}\left(\mathbb{E}_{1}\right)$ and $L_{h}^{2}\left(\mathbb{E}_{2}\right)$ [itself a dense subset of the hilbert space product $L_{h}^{2}\left(\mathbb{E}_{1}\right) \otimes L_{h}^{2}\left(\mathbb{E}_{2}\right)$ ] into $L_{h}^{2}(\mathbb{E})$. Let us show that $\beta_{0}$ is an isometry onto a dense subset.

Firstly if $\langle$,$\rangle is the hilbert space scalar product then$

$$
\begin{aligned}
& \left\langle\psi_{1} \otimes \psi_{2}, \phi_{1} \otimes \phi_{2}\right\rangle=\left\langle\psi_{1}, \phi_{1}\right\rangle\left\langle\psi_{2}, \phi_{2}\right\rangle \\
& \quad=\int_{E_{1}} \overline{\psi_{1}\left(X_{1}\right)} \phi_{1}\left(X_{1}\right) d \bar{\mu}_{1}\left(X_{1}\right) \int_{E_{2}} \overline{\psi_{2}\left(X_{2}\right)} \phi_{2}\left(X_{2}\right) d \bar{\mu}_{2}\left(X_{2}\right)
\end{aligned}
$$

while by (B9)

$$
\left\langle\beta_{0}\left(\psi_{1} \otimes \psi_{2}\right), \beta_{0}\left(\phi_{1} \otimes \phi_{2}\right)\right\rangle=\int_{E} \overline{\psi_{1}\left(X_{1}\right) \psi_{2}\left(X_{2}\right)} \phi_{1}\left(X_{1}\right) \phi_{2}\left(X_{2}\right) d \bar{\mu}\left(X_{1} \otimes X_{2}\right) .
$$

The homogeneity of the $\psi$ 's and $\phi$ 's reduces the integrals to ones over $M_{1} \times M_{2}$, whence their equality because $d \mu\left(x_{1}, x_{2}\right)=d \mu_{1}\left(x_{1}\right) d \mu_{2}\left(x_{2}\right)$. Hence $\beta_{0}$ is an isometry.

Secondly the question whether the image of $\beta_{0}$ is dense in $L_{h}^{2}(\mathbb{E})$ can be studied in a coordinate patch, and there a favorable answer would follow from the full result (i.e. Fact B14 itself) formulated for trivial gauge-spaces. To establish the latter we need only write

$$
\begin{aligned}
L_{h}^{2}\left(\left(C \times M_{1}\right) \otimes\left(C \times M_{2}\right)\right) & =L_{h}^{2}\left(C \times M_{1} \times M_{2}\right)=L^{2}\left(M_{1} \times M_{2}\right) \\
& =L^{2}\left(M_{1}\right) \otimes L^{2}\left(M_{2}\right)=L_{h}^{2}\left(C \times M_{1}\right) \otimes L_{h}^{2}\left(C \times M_{2}\right),
\end{aligned}
$$

where the first isomorphism follows from Facts B6(i) and B3, the second and fourth are obvious [see (4.9), (4.10)], and the third is well known.

It follows that $\beta_{0}$ extends to an isometry

$$
\beta: L_{h}^{2}\left(\mathbb{E}_{1}\right) \otimes L_{h}^{2}\left(\mathbb{E}_{2}\right) \rightarrow L_{h}^{2}(\mathbb{E}) .
$$

Just as a gauge-space (with volume element) $\mathbb{E}$ gives rise to a hilbert space $L_{h}^{2}(\mathbb{E})$, a (volume preserving) realization $R$ on $\mathbb{E}$ gives rise, via the natural prescription

$$
\left[L_{h}^{2}(R)(g)\right] \cdot \psi=\psi \circ R\left(g^{-1}\right),
$$


to a unitary representation $L_{h}^{2}(R)$, acting in $L_{h}^{2}(\mathbb{E})$. [In the body of the paper this process was used to derive $\boldsymbol{\Lambda}=L_{h}^{2}(\Lambda), \boldsymbol{\Pi}=L_{h}^{2}(\Pi)$, etc.] As for the spaces, we have also for the realizations,

Fact B 15. If $R_{1}$ and $R_{2}$ are volume preserving realizations of $G$ in $\mathbb{E}_{1}$ and $\mathbb{E}_{2}$ respectively, then under the isomorphism of Fact B14

$$
L_{h}^{2}\left(R_{1} \otimes R_{2}\right) \simeq L_{h}^{2}\left(R_{1}\right) \otimes L_{h}^{2}\left(R_{2}\right) .
$$

Proof. Let $\beta$ be the isometry constructed in the last proof. We must show, for each $g \in G$,

$$
\beta \circ\left[L_{h}^{2}\left(R_{1}\right)(g) \otimes L_{h}^{2}\left(R_{2}\right)(g)\right]=L_{h}^{2}\left(R_{1} \otimes R_{2}\right)(g) \circ \beta .
$$

Applying this to an arbitrary $\psi_{1} \otimes \psi_{2}$ (and suppressing $g$ ) reduces it to

$$
\beta\left(\psi_{1} \circ R_{1} \otimes \psi_{2} \circ R_{2}\right)=\beta\left(\psi_{1} \otimes \psi_{2}\right) \circ\left(R_{1} \otimes R_{2}\right)
$$

which itself, when applied to an arbitrary $X_{1} \otimes X_{2} \in E_{1} \otimes E_{2}$ reduces to $\psi_{1} \circ R_{1}\left(X_{1}\right) \psi_{2} \circ R_{2}\left(X_{2}\right)=\beta\left(\psi_{1} \otimes \psi_{2}\right)\left(R_{1}\left(X_{1}\right) \otimes R_{2}\left(X_{2}\right)\right)$, which - at last - is immediate from the definition of $\beta$.

\section{References}

1. Friedman, J.L., Sorkin, R.D. : Phys. Rev. D20, 2511 (1979)

2. Barut, A.O.: Phys. Rev. D10, 2712 (1974)

3. Goldhaber, A.S. : Phys. Rev. Lett. 36, 1122 (1976)

4. Souriau, J.-M.: Structure des systèmes dynamiques. Paris: Dunod 1970

5. Kostant, B.: Lecture Notes in Mathematics 170. Berlin, Heidelberg, New York: Springer 1970

6. Trautman, A.: Intern. J. Theor. Phys. 16, 561 (1977)

7. Lubkin, E.: Ann. Phys. 23, 233 (1963)

8. Wu, T.T., Yang, C.N.: Phys. Rev. D12, 3845 (1975)

9. Tolkachev, E.A., Tomil'chik: Phys. Lett. 81 B, 173 (1979)

Communicated by R. Geroch 